\title{
Bounds and extremal graphs of second reformulated Zagreb index for graphs with cyclomatic number at most three
}

\author{
Abhay Rajpoot, Lavanya Selvaganesh* \\ Dept. of Mathematical Sciences \\ Indian Institute of Technology (BHU) \\ Varanasi-221005, INDIA \\ *Corresponding author: lavanyas.mat@iitbhu.ac.in
}

\begin{abstract}
Miličević et al., in 2004, introduced topological indices known as Reformulated Zagreb indices, where they modified Zagreb indices using the edge-degree instead of vertex degree. In this paper, we present a simple approach to find the upper and lower bounds of the second reformulated Zagreb index, $E M_{2}(G)$, by using six graph operations/transformations. We prove that these operations significantly alter the value of reformulated Zagreb index. We apply these transformations and identify those graphs with cyclomatic number at most 3, namely trees, unicyclic, bicyclic and tricyclic graphs, which attain the upper and lower bounds of second reformulated Zagreb index for graphs.
\end{abstract}

Keywords: Bicyclic graphs; trees; tricyclic graphs; unicyclic graphs; reformulated Zagreb index. Mathematics Subject Classification (2010): 05C05; 05C07; 05C92

\section{Introduction}

Topological indices play a crucial role in characterizing the properties of molecules in terms of physical, chemical and biological. Several types of topological indices exist in mathematical chemistry, such as the Wiener index (Wiener, 1947); Hyper Wiener index (Klein et al., 1995); Hosoya index (Hosoya, 1971); Randić index (Randić, 1975); Augmented Zagreb index (Furtula et al., 2010); Harmonic index (Fajtlowicz, 1987); to name a few. For more details on topological indices, we refer to some articles here (Basak, 2016; Devillers \& Balaban, 2000; Gutman, 2013; Gutman et al., 2014, 2020; Karelson, 2000; Narayankar et al., 2020; Rada \& Bermudo, 2019; Romero-Valencia et al., 2019; Shang, 2016; Varmuza et al., 2012).

Throughout this paper, we consider only non trivial simple connected graphs. Let a simple connected graph of $n$ vertices be denoted by $G=(V(G), E(G))$, where $V(G)$ and $E(G)$ denote the set of vertices and set of edges of the graph respectively. Let $d_{G}(u)$ denote the degree of the vertex $u, d_{G}(e)$ denotes the degree of the edge e. $N_{G}(u)=\{v \in V(G): u v \in E(G)\}$ denotes the set of all neighbours of vertex $u$ in $G$, that is, $d_{G}(u)=\left|N_{G}(u)\right|$. The cyclomatic number of a graph is defined as $c y(G)=|E(G)|-|V(G)|$ +1 . Trees have no cycles and hence has cyclomatic number to be 0 . When $m=n$, $G$ has cyclomatic number 1 and called as a unicyclic graph. When $m=n+1, G$ has cyclomatic number 2 and referred to as bicyclic graph. When $m=n+2, G$ has cyclomatic number 3 and referred to as triyclic graph. Let $S_{n}, P_{n}$, and $C_{n}$ denoted the star graph, path graph, and cycle graph of $n$ vertices. Other notations and definitions are taken from the book (Bondy et al., 1976).

The Zagreb indices are one of the most important and well studied topological indices in the literature. It first appeared in 1972, during the study of $\pi$-energy of conjugate molecules. For a simple graph $G$, Zagreb indices (Gutman \& Das , 2004; Gutman \& Trinajstić , 1972) are defined as 


$$
M_{1}(G)=\sum_{u \in V(G)} d_{G}^{2}(u), \quad M_{2}(G)=\sum_{u v \in E(G)} d_{G}(u) \cdot d_{G}(v),
$$

where $d_{G}(u)$ denotes the degree of the vertex $u \in V(G)$.

Motivated by the concept of line graph $L(G)$ of a graph $G$, where the edges in $G$ represents the vertices of line graph $L(G)$, Miličević et al. (Miličević et al., 2004) introduced a pair of topological indices in terms of edge-degree instead of vertex degree in Zagreb index and called it as Reformulated Zagreb index. For the simple graph $G$, first and second Reformulated Zagreb indices are defined as

$$
E M_{1}(G)=\sum_{e \in E(G)} d_{G}^{2}(e), \quad E M_{2}(G)=\sum_{e \sim f} d_{G}(e) \cdot d_{G}(f),
$$

where $e \sim f$ means the edge $e$ and $f$ are adjacent in graph $G$, i.e., the edge $e$ and $f$ have a common vertex. Further, if $e=u v$, then note that $d_{G}(e)=d_{G}(u)+d_{G}(v)-2$.

Nowadays many researchers are interested in finding the bounds of topological indices of graphs, as well as characterizing the extremal graphs, see articles (Ali, Elumalai \& Mansour, 2020; Ali, Milovanović, Matejić \& Milovanović, 2020; Ali et al., 2019; Borovicanin et al., 2017; Deng, 2007; Ghalavand \& Ashrafi, 2020; Gutman, 2013; Khalifeh et al., 2009; Rajpoot \& Selvaganesh, 2020; Xu \& Das, 2012; Noureen et al., 2020; Aouchiche et al., 2020; Ali et al., 2016; Ali \& Bhatti, 2016). For the bounds of reformulated Zagreb indices we refer to (De, 2012; Ghalavand \& Ashrafi, 2017; Ilić \& Zhou, 2012; Ji et al., 2014, 2015; Su et al., 2011). In (Deng, 2007), the author proposes few graph operations which are useful in finding the bounds for Zagreb indices while in (Ji et al., 2014, 2015) the authors discusses some graph operations to study the bounds of first reformulated Zagreb index.

In this paper, we focus on finding the bounds of second reformulated Zagreb index. In particular, we give the bounds for special graph classes, namely the trees, unicyclic graphs, bicyclic graphs and tricyclic graphs. In order to study the bounds for graphs, we propose to use certain transformations which help us in obtaining required results. Further, we also find the extremal graphs which attain these bounds.

The organization of the paper is as follows: In Section 2, we give three transformations which increases the value of the second reformulated Zagreb index. In Section 3, we give three transformations which decrease the value of $E M_{2}$. In Section 4, we compute the bounds(both upper and lower) for $E M_{2}(G)$ when $G$ is a tree, a unicyclic graph, a bicyclic graph, and a tricyclic graph. In this process, we also find the graphs which attain these bounds.

\section{Three graph transformations which increase the second reformulated Zagreb index}

In this section, we discuss three graph operations as defined in (Deng, 2007; Ji et al., 2014) and show how they impact the second reformulated Zagreb index.

Transformation A: Let $G_{0}$ be a non-trivial graph and $u$ be any vertex of $G_{0}$. Let $G_{1}$ be a graph obtained from $G_{0}$ by joining $u$ to the center of a star such that $d_{G_{1}}(v) \geq 2$, and $\left\{w_{1}, w_{2}, \ldots, w_{t}\right\}$ is the pendant neighbour of $v$ in $G_{1}$, see Figure 1 . Here, we say that $G_{2}$ is obtained from $G_{1}$ by transformation $\mathbf{A}$ and write as $G_{2}=G_{1}-\left\{v w_{1}, v w_{2}, \ldots, v w_{t}\right\}+\left\{u w_{1}, u w_{2}, \ldots, u w_{t}\right\}$.

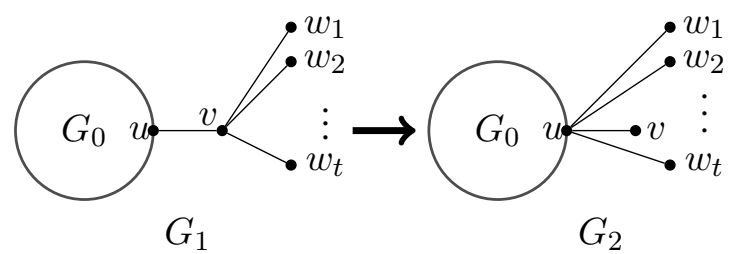

Fig. 1. Representation of transformation $A$.

Lemma 2.1 If $G_{2}$ is obtained by applying transformation $A$ on $G_{1}$, then $E M_{2}\left(G_{1}\right)<E M_{2}\left(G_{2}\right)$. 
Proof: Let $N_{G_{0}}(u)=\left\{x \in V\left(G_{0}\right): u x \in E\left(G_{0}\right)\right\}$. From the definition of $E M_{2}(G)$, we have

$$
\begin{aligned}
E M_{2}\left(G_{1}\right)= & \sum_{e_{i}, e_{j} \in E\left(G_{0} \backslash\{u\}\right)} d_{G_{1}}\left(e_{i}\right) d_{G_{1}}\left(e_{j}\right)+\sum_{x \in N_{G_{0}}(u)} \sum_{y \in N_{G_{0}}(x) \backslash\{u\}} d_{G_{1}}(u x) d_{G_{1}}(x y) \\
& +\sum_{x, y \in N_{G_{0}}(u)} d_{G_{1}}(x u) d_{G_{1}}(u y)+\sum_{x \in N_{G_{0}}(u)} d_{G_{1}}(x u) d_{G_{1}}(u v)+\sum_{i=1}^{t} d_{G_{1}}(u v) d_{G_{1}}\left(v w_{i}\right) \\
& +\frac{t(t-1)}{2}\left(d_{G_{1}}(v)-1\right)^{2},
\end{aligned}
$$

and

$$
\begin{aligned}
E M_{2}\left(G_{2}\right)= & \sum_{e_{i}, e_{j} \in E\left(G_{0} \backslash\{u\}\right)} d_{G_{1}}\left(e_{i}\right) d_{G_{1}}\left(e_{j}\right)+\sum_{x \in N_{G_{0}}(u)} \sum_{y \in N_{G_{0}}(x) \backslash\{u\}}\left(d_{G_{1}}(u x)+t\right) d_{G_{1}}(x y) \\
& +\sum_{x, y \in N_{G_{0}}(u)}\left(d_{G_{1}}(x u)+t\right)\left(d_{G_{1}}(u y)+t\right)+\sum_{x \in N_{G_{0}}(u)}\left(d_{G_{1}}(x u)+t\right) d_{G_{1}}(u v) \\
& +t \sum_{x \in N_{G_{0}}(u)}\left(d_{G_{1}}(x u)+t\right)\left(d_{G_{1}}(u)+t-1\right)+\frac{t(t-1)}{2}\left(d_{G_{1}}(u)+t-1\right)^{2} \\
& +t\left(d_{G_{1}}(u)+t-1\right)^{2} .
\end{aligned}
$$

Then,

$$
\begin{aligned}
E M_{2}\left(G_{2}\right)-E M_{2}\left(G_{1}\right)= & \sum_{x \in N_{G_{0}}(u)} \sum_{y \in N_{G_{0}}(x) \backslash\{u\}} t d_{G_{1}}(x y)+\sum_{x, y \in N_{G_{0}}(u)} t\left(d_{G_{1}}(x u)+d_{G_{1}}(u y)+t\right) \\
& +\sum_{x \in N_{G_{0}}(u)} t\left(d_{G_{1}}(u)+t-1\right)\left(d_{G_{1}}(x u)+t+1\right)-t^{2}\left(d_{G_{1}}(u)+t-1\right) \\
& +\frac{t(t-1)}{2}\left(\left(d_{G_{1}}(u)+2 t-1\right)\left(d_{G_{1}}(u)-1\right)\right)+t\left(d_{G_{1}}(u)+t-1\right)^{2} \\
& \geq t\left(d_{G_{1}}(u)+t-1\right)^{2} \\
& >0 .
\end{aligned}
$$

Transformation B: Let $u$ and $v$ be any two vertices of a simple connected graph $G_{1}$ such that there is a path of length $t \geq 1, P_{t}=u_{0}(=u), u_{1}, \ldots, u_{t-1}, u_{t}(=v)$ which connect the vertices $u$ and $v$, see Figure 2. Here, we say that $G_{2}$ is obtained from $G_{1}$ by transformation $\mathbf{B}$ and write as $G_{2}=$ $G_{1}-\left\{u_{0} u_{1}, u_{1} u_{2}, \ldots u_{t-1} u_{t}\right\}+\left\{w u_{1}, w u_{2}, \ldots, w u_{t}\right\}$, where $w=u \circ v$.

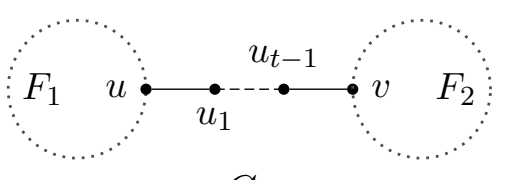

$G_{1}$

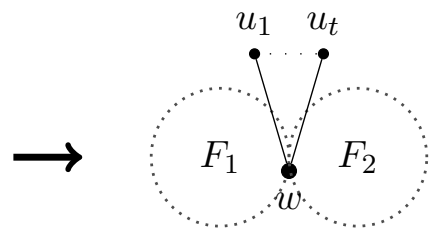

$G_{2}$

Fig. 2. Representation of transformation $B$.

Lemma 2.2 If $G_{2}$ is obtained from $G_{1}$ using transformation $B$, then $E M_{2}\left(G_{1}\right)<E M_{2}\left(G_{2}\right)$.

Proof: Let $d_{G_{1}}(u)=a+1$, and $d_{G_{1}}(v)=b+1$, where $a \geq 1, b \geq 1$. Then the degree of edges $d_{G_{1}}\left(u u_{1}\right)=a+1, d_{G_{1}}\left(v u_{t-1}\right)=b+1$, and $d_{G_{2}}\left(w u_{i}\right)=(a+b+t-1)$, for $i=1$ to $t$. First we assume 
that $t \geq 3$, then from the definition of $E M_{2}(G)$, we have

$$
\begin{aligned}
& E M_{2}\left(G_{1}\right)=\sum_{e_{i}, e_{j} \in E\left(F_{1} \backslash\{u\}\right)} d_{G_{1}}\left(e_{i}\right) d_{G_{1}}\left(e_{j}\right)+\sum_{e_{i}, e_{j} \in E\left(F_{2} \backslash\{v\}\right)} d_{G_{1}}\left(e_{i}\right) d_{G_{1}}\left(e_{j}\right) \\
& +\sum_{x \in N_{F_{1}}(u)} \sum_{y \in N_{F_{1}}(x) \backslash\{u\}} d_{G_{1}}(u x) d_{G_{1}}(x y)+\sum_{x \in N_{F_{2}}(v)} \sum_{y \in N_{F_{2}}(x) \backslash\{v\}} d_{G_{1}}(v x) d_{G_{1}}(x y) \\
& +\sum_{x, y \in N_{F_{1}}(u)} d_{G_{1}}(x u) d_{G_{1}}(u y)+\sum_{x, y \in N_{F_{2}}(v)} d_{G_{1}}(x v) d_{G_{1}}(v y) \\
& +\sum_{x \in N_{F_{1}}(u)} d_{G_{1}}(x u) d_{G_{1}}\left(u u_{1}\right)+\sum_{x \in N_{F_{2}}(v)} d_{G_{1}}(x v) d_{G_{1}}\left(v u_{t-1}\right) \\
& +2 d_{G_{1}}\left(u u_{1}\right)+2 d_{G_{1}}\left(u_{t-1} v\right)+4(t-3) . \\
& E M_{2}\left(G_{2}\right)=\sum_{e_{i}, e_{j} \in E\left(F_{1} \backslash\{w\}\right)} d_{G_{1}}\left(e_{i}\right) d_{G_{1}}\left(e_{j}\right)+\sum_{e_{i}, e_{j} \in E\left(F_{2} \backslash\{w\}\right)} d_{G_{1}}\left(e_{i}\right) d_{G_{1}}\left(e_{j}\right) \\
& +\sum_{x \in N_{F_{1}}(w)} \sum_{y \in N_{F_{1}}(x) \backslash\{w\}} d_{G_{2}}(w x) d_{G_{1}}(x y)+\sum_{x \in N_{F_{2}}(w)} \sum_{y \in N_{F_{2}}(x) \backslash\{w\}} d_{G_{2}}(w x) d_{G_{1}}(x y) \\
& +\sum_{x, y \in N_{F_{1}}(w)} d_{G_{2}}(x w) d_{G_{2}}(w y)+\sum_{x, y \in N_{F_{2}}(w)} d_{G_{2}}(x w) d_{G_{2}}(w y) \\
& +t \sum_{x \in N_{F_{1}}(w)} d_{G_{2}}(x w)\left(d_{G_{2}}(w)-1\right)+t \sum_{x \in N_{F_{2}}(w)} d_{G_{2}}(x w)\left(d_{G_{2}}(w)-1\right) \\
& +\sum_{x \in N_{F_{1}}(w)} \sum_{y \in N_{F_{2}}(w)} d_{G_{2}}(x w) d_{G_{2}}(w y)+\frac{1}{2} t(t-1)\left(d_{G_{2}}(w)-1\right)^{2},
\end{aligned}
$$

since $d_{G_{2}}(w)=d_{G_{1}}(u)+d_{G_{1}}(v)+t-2, N_{F_{1}}(w)=N_{F_{1}}(u)$ and $N_{F_{2}}(w)=N_{F_{2}}(v)$. Then

$$
\begin{aligned}
E M_{2}\left(G_{2}\right)-E M_{2}\left(G_{1}\right)> & \sum_{x \in N_{F_{1}}(w)} \sum_{y \in N_{F_{2}}(w)} d_{G_{2}}(x w) d_{G_{2}}(w y)+\frac{1}{2} t(t-1)\left(d_{G_{2}}(w)-1\right)^{2} \\
& -2 d_{G_{1}}\left(u u_{1}\right)-2 d_{G_{1}}\left(u_{t-1} v\right)-4(t-3) \\
> & (a+b+t-1)^{2}-2(a+1)-2(b+1)-4(t-3) \\
\geq & 2(a+1)(b+1) \\
> & 0 .
\end{aligned}
$$

Similarly proof holds for the remaining values of $t$, that is, $t=1$ or $t=2$. We omit the proofs for brevity.

Transformation C: Suppose $u$ and $v$ are any two vertices of a simple connected graph $G_{0}$. Let $G_{1}$ be the graph obtained from $G_{0}$ by adding pendant vertices $\left\{v_{1}, v_{2}, \ldots, v_{t}\right\},\left\{u_{1}, u_{2}, \ldots, u_{r}\right\}$ at $v$ and $u$ respectively as shown in Figure 3. Now we have two possibilities for a transformation namely, $G_{2}=G_{1}-$ $\left\{u u_{1}, u u_{2}, \ldots, u u_{r}\right\}+\left\{v u_{1}, v u_{2}, \ldots, v u_{r}\right\}$, and $G_{3}=G_{1}-\left\{v v_{1}, v v_{2}, \ldots, v v_{t}\right\}+\left\{u v_{1}, u v_{2}, \ldots, u v_{t}\right\}$. Here, we say that $G_{2}$ and $G_{3}$ are obtained from $G_{1}$ by transformation $\mathbf{C}$.

Lemma 2.3 If graphs $G_{2}$ and $G_{3}$ are obtained from graph $G_{1}$ by transformation $C$, then either $E M_{2}\left(G_{1}\right)<E M_{2}\left(G_{2}\right)$ or $E M_{2}\left(G_{1}\right)<E M_{2}\left(G_{3}\right)$.

Proof: Let $d_{G_{0}}(u)=p, d_{G_{0}}(v)=q$. Without loss of generality, we assume that $d_{G_{0}}(v) \leq d_{G_{0}}(u)$, let $p=q+a$, where $a \geq 0$. Now, we prove this lemma by making conditions on the vertices $u$ and $v$. 

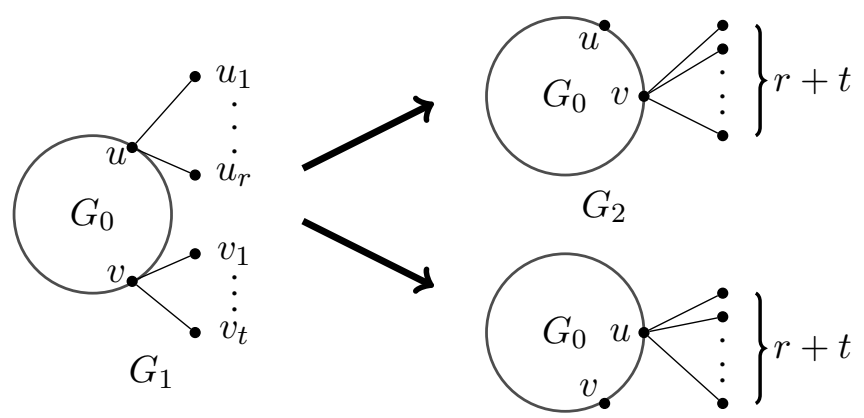

$G_{3}$

Fig. 3. Representation of transformation $C$.

Case 1: If vertex $u$ and $v$ are not adjacent. Then, we have two subcases.

Subcase 1.1: If $N_{G_{0}}(u) \cap N_{G_{0}}(v)=\emptyset$, then from the definition of $E M_{2}(G)$, we have

$$
\begin{aligned}
& E M_{2}\left(G_{1}\right)=\sum_{e_{i}, e_{j} \in E\left(G_{0} \backslash\{u, v\}\right)} d_{G_{1}}\left(e_{i}\right) d_{G_{1}}\left(e_{j}\right)+\sum_{x \in N_{G_{0}}(u)} \sum_{y \in N_{G_{0}}(x) \backslash\{u\}} d_{G_{1}}(u x) d_{G_{1}}(x y) \\
& +\sum_{x, y \in N_{G_{0}}(u)} d_{G_{1}}(x u) d_{G_{1}}(u y)+\sum_{x \in N_{G_{0}}(v)} \sum_{y \in N_{G_{0}}(x) \backslash\{v\}} d_{G_{1}}(v x) d_{G_{1}}(x y) \\
& +\sum_{x, y \in N_{G_{0}}(v)} d_{G_{1}}(x v) d_{G_{1}}(v y)+r(q+a+r-1) \sum_{x \in N_{G_{0}}(u)} d_{G_{1}}(x u) \\
& +t(q+t-1) \sum_{x \in N_{G_{0}}(v)} d_{G_{1}}(x v)+\frac{r(r-1)}{2}(q+a+r-1)^{2} \\
& \quad+\frac{t(t-1)}{2}(q+t-1)^{2}, \\
& \quad M_{2}\left(G_{2}\right)=\sum_{e_{i}, e_{j} \in E\left(G_{0} \backslash\{u, v\}\right)} d_{G_{1}}\left(e_{i}\right) d_{G_{1}}\left(e_{j}\right)+\sum_{x \in N_{G_{0}}(u)} \sum_{y \in N_{G_{0}}(x) \backslash\{u\}}\left(d_{G_{1}}(u x)-r\right) d_{G_{1}}(x y) \\
& \left.+\sum_{x, y \in N_{G_{0}}(u)}\left(d_{G_{1}}(x u)-r\right)\left(d_{G_{1}}(u y)-r\right)+\sum_{x \in N_{G_{0}}(v)}(v x)+r\right) d_{G_{1}}(x y) \\
& +\sum_{x, y \in N_{G_{0}}(v)}\left(d_{G_{1}}(x v)+r\right)\left(d_{G_{1}}(x) \backslash\{v\}\right. \\
& \left.+\frac{(r+t)(r+t-1)}{2}(q)+r+t-1\right)^{2}, \quad(r+t)(q+r+t-1) \sum_{x \in N_{G_{0}}(v)}\left(d_{G_{1}}(x v)+r\right)
\end{aligned}
$$

and

$$
\begin{aligned}
& E M_{2}\left(G_{3}\right)=\sum_{e_{i}, e_{j} \in E\left(G_{0} \backslash\{u, v\}\right)} d_{G_{1}}\left(e_{i}\right) d_{G_{1}}\left(e_{j}\right)+\sum_{x \in N_{G_{0}}(u)} \sum_{y \in N_{G_{0}}(x) \backslash\{u\}}\left(d_{G_{1}}(u x)+t\right) d_{G_{1}}(x y) \\
& \quad+\sum_{x, y \in N_{G_{0}}(u)}\left(d_{G_{1}}(x u)+t\right)\left(d_{G_{1}}(u y)+t\right)+\sum_{x \in N_{G_{0}}(v)} \sum_{y \in N_{G_{0}}(x) \backslash\{v\}}\left(d_{G_{1}}(v x)-t\right) d_{G_{1}}(x y) \\
& \quad+\sum_{x, y \in N_{G_{0}}(v)}\left(d_{G_{1}}(x v)-t\right)\left(d_{G_{1}}(v y)-t\right)+\frac{(r+t)(r+t-1)}{2}(q+a+r+t-1)^{2} \\
& \quad+(r+t)(q+a+r+t-1) \sum_{x \in N_{G_{0}}(u)}\left(d_{G_{1}}(x u)+t\right) .
\end{aligned}
$$


Then,

$$
\begin{aligned}
E M_{2}\left(G_{2}\right) & -E M_{2}\left(G_{1}\right)=-r \sum_{x \in N_{G_{0}}(u)} \sum_{y \in N_{G_{0}}(x) \backslash\{u\}} d_{G_{1}}(x y)-r \sum_{x, y \in N_{G_{0}}(u)}\left(d_{G_{1}}(x u)+d_{G_{1}}(u y)-r\right) \\
& -r(q+r+a-1) \sum_{x \in N_{G_{0}}(u)} d_{G_{1}}(x u)+r \sum_{x \in N_{G_{0}}(v)} \sum_{y \in N_{G_{0}}(x) \backslash\{v\}} d_{G_{1}}(x y) \\
& +r \sum_{x, y \in N_{G_{0}}(v)}\left(d_{G_{1}}(x v)+d_{G_{1}}(v y)+r\right)+r(q+r+2 t-1) \sum_{x \in N_{G_{0}}(v)} d_{G_{1}}(x v) \\
& +q r(r+t)(q+r+t-1)+r t(q+r+t-1)^{2}+\frac{1}{2} r t(t-1)(2 q+r+2 t-2) \\
& +\frac{1}{2} r(r-1)(t-a)(2 q+2 r+t+a-2),
\end{aligned}
$$

and

$$
\begin{aligned}
E M_{2}\left(G_{3}\right)- & E M_{2}\left(G_{1}\right)=t \sum_{x \in N_{G_{0}}(u)} \sum_{y \in N_{G_{0}}(x) \backslash\{u\}} d_{G_{1}}(x y)+t \sum_{x, y \in N_{G_{0}}(u)}\left(d_{G_{1}}(x u)+d_{G_{1}}(u y)+t\right) \\
& +t(q+2 r+t+a-1) \sum_{x \in N_{G_{0}}(u)} d_{G_{1}}(x u)-t \sum_{x \in N_{G_{0}}(v)} \sum_{y \in N_{G_{0}}(x) \backslash\{v\}} d_{G_{1}}(x y) \\
& -t \sum_{x, y \in N_{G_{0}}(v)}\left(d_{G_{1}}(x v)+d_{G_{1}}(v y)-t\right)-t(q+t-1) \sum_{x \in N_{G_{0}}(v)} d_{G_{1}}(x v) \\
& +t(q+a)(r+t)(q+r+t+a-1)+\frac{1}{2} r t(r-1)(2 q+2 r+t+2 a-2) \\
& +r t(q+r+t+a-1)^{2}+\frac{1}{2} t(t-1)(r+a)(2 q+r+2 t+a-2) .
\end{aligned}
$$

Note that if either of the expressions (1) or (2) is greater than 0, then the lemma holds. Suppose $E M_{2}\left(G_{2}\right)-E M_{2}\left(G_{1}\right) \leq 0$, then we will show that $E M_{2}\left(G_{3}\right)-E M_{2}\left(G_{1}\right)$ has to be greater than 0 . We will now find a relation from Equation (1) by letting $E M_{2}\left(G_{2}\right)-E M_{2}\left(G_{1}\right) \leq 0$ and use the same to prove our claim. That is, we get

$$
\begin{aligned}
& q(r+t)(q+r+t-1)+t(q+r+t-1)^{2}+\frac{1}{2}(r-1)(t-a)(2 q+2 r+t+a-2) \\
& +\frac{1}{2} t(t-1)(2 q+r+2 t-2) \\
& \leq \sum_{x \in N_{G_{0}}(u)} \sum_{y \in N_{G_{0}}(x) \backslash\{u\}} d_{G_{1}}(x y)+\sum_{x, y \in N_{G_{0}}(u)}\left(d_{G_{1}}(x u)+d_{G_{1}}(u y)-r\right) \\
& +(q+r+a-1) \sum_{x \in N_{G_{0}}(u)} d_{G_{1}}(x u)-\sum_{x \in N_{G_{0}}} \sum_{y \in N_{G_{0}}(x) \backslash\{v\}} d_{G_{1}}(x y) \\
& -\sum_{x, y \in N_{G_{0}}(v)}\left(d_{G_{1}}(x v)+d_{G_{1}}(v y)+r\right)-(q+r+2 t-1) \sum_{x \in N_{G_{0}}(v)} d_{G_{1}}(x v) \\
& \leq \sum_{x \in N_{G_{0}}(u)} d_{y \in N_{G_{0}}(x) \backslash\{u\}} d_{G_{1}}(x y)+\sum_{x, y \in N_{G_{0}}(u)}\left(d_{G_{1}}(x u)+d_{G_{1}}(u y)+t\right) \\
& \quad+(q+2 r+t+a-1) \sum_{x \in N_{G_{0}}(u)} d_{G_{1}}(x u)-\sum_{x \in N_{G_{0}}(v)} \sum_{y \in N_{G_{0}}(x) \backslash\{v\}} d_{G_{1}}(x y) \\
& \quad-\sum_{x, y \in N_{G_{0}}(v)}\left(d_{G_{1}}(x v)+d_{G_{1}}(v y)-t\right)-(q+t-1) \sum_{x \in N_{G_{0}}(v)} d_{G_{1}}(x v) .
\end{aligned}
$$


Substituting for the first six terms of right hand side of equation (2) with the inequality (3), we have

$$
\begin{aligned}
& E M_{2}\left(G_{3}\right)-E M_{2}\left(G_{1}\right) \geq t\left\{q(r+t)(q+r+t-1)+\frac{1}{2}(r-1)(t-a)(2 q+2 r+t+a-2)\right. \\
& +(q+a)(r+t)(q+r+t+a-1)+\frac{1}{2} t(t-1)(2 q+r+2 t-2)+t(q+r+t-1)^{2} \\
& +\frac{1}{2} r(r-1)(2 q+2 r+t+2 a-2)+\frac{1}{2}(t-1)(r+a)(2 q+r+2 t+a-2) \\
& \left.+r(q+r+t+a-1)^{2}\right\} \\
& >0, \text { since } q \geq 2, \text { and } r, t \geq 1 .
\end{aligned}
$$

Subcase 1.2: If $N_{G_{0}}(u) \cap N_{G_{0}}(v) \neq \emptyset$, then from the definition of $E M_{2}(G)$, we have

$$
\begin{aligned}
& E M_{2}\left(G_{1}\right)=\sum_{e_{i}, e_{j} \in E\left(G_{0} \backslash\{u, v\}\right)} d_{G_{1}}\left(e_{i}\right) d_{G_{1}}\left(e_{j}\right)+\sum_{x \in N_{G_{0}}(u) \backslash N_{G_{0}}(v)} \sum_{y \in N_{G_{0}}(x) \backslash\{u\}} d_{G_{1}}(u x) d_{G_{1}}(x y) \\
& +\sum_{x, y \in N_{G_{0}}(u)} d_{G_{1}}(x u) d_{G_{1}}(u y)+\sum_{x, y \in N_{G_{0}}(v)} d_{G_{1}}(x v) d_{G_{1}}(v y)+r(q+a+r-1) \sum_{x \in N_{G_{0}}(u)} d_{G_{1}}(x u) \\
& +t(q+t-1) \sum_{x \in N_{G_{0}}(v)} d_{G_{1}}(x v)+\sum_{x \in N_{G_{0}}(v) \backslash N_{G_{0}}(u)} \sum_{y \in N_{G_{0}}(x) \backslash\{v\}} d_{G_{1}}(v x) d_{G_{1}}(x y) \\
& +\sum_{x \in N_{G_{0}}(u) \cap N_{G_{0}}(v)} d_{G_{1}}(u x) d_{G_{1}}(x v)+\frac{r(r-1)}{2}(q+a+r-1)^{2}+\frac{t(t-1)}{2}(q+t-1)^{2}, \\
& E M_{2}\left(G_{2}\right)=\sum_{e_{i}, e_{j} \in E\left(G_{0} \backslash\{u, v\}\right)} d_{G_{1}}\left(e_{i}\right) d_{G_{1}}\left(e_{j}\right)+\sum_{x \in N_{G_{0}}(u) \backslash N_{G_{0}}(v)} \sum_{y \in N_{G_{0}}(x) \backslash\{u\}}\left(d_{G_{1}}(u x)-r\right) d_{G_{1}}(x y) \\
& +\sum_{x, y \in N_{G_{0}}(u)}\left(d_{G_{1}}(x u)-r\right)\left(d_{G_{1}}(u y)-r\right)+\sum_{x, y \in N_{G_{0}}(v)}\left(d_{G_{1}}(x v)+r\right)\left(d_{G_{1}}(v y)+r\right) \\
& +\sum_{x \in N_{G_{0}}(v) \backslash N_{G_{0}}(u)} \sum_{y \in N_{G_{0}}(x) \backslash\{v\}}\left(d_{G_{1}}(v x)+r\right) d_{G_{1}}(x y)+\sum_{x \in N_{G_{0}}(u) \cap N_{G_{0}}(v)}\left(d_{G_{1}}(u x)-r\right)\left(d_{G_{1}}(x v)+r\right) \\
& +(r+t)(q+r+t-1) \sum_{x \in N_{G_{0}}(v)}\left(d_{G_{1}}(x v)+r\right)+\frac{(r+t)(r+t-1)}{2}(q+r+t-1)^{2}, \\
& \text { and } E M_{2}\left(G_{3}\right)=\sum_{e_{i}, e_{j} \in E\left(G_{0} \backslash\{u, v\}\right)} d_{G_{1}}\left(e_{i}\right) d_{G_{1}}\left(e_{j}\right)+\sum_{x \in N_{G_{0}}(u) \backslash N_{G_{0}}(v)} \sum_{y \in N_{G_{0}}(x) \backslash\{u\}}\left(d_{G_{1}}(u x)+t\right) d_{G_{1}}(x y) \\
& +\sum_{x, y \in N_{G_{0}}(u)}\left(d_{G_{1}}(x u)+t\right)\left(d_{G_{1}}(u y)+t\right)+(r+t)(q+a+r+t-1) \sum_{x \in N_{G_{0}}(u)}\left(d_{G_{1}}(x u)+t\right) \\
& +\sum_{x \in N_{G_{0}}(v) \backslash N_{G_{0}}(u)} \sum_{y \in N_{G_{0}}(x) \backslash\{v\}}\left(d_{G_{1}}(v x)-t\right) d_{G_{1}}(x y)+\sum_{x, y \in N_{G_{0}}(v)}\left(d_{G_{1}}(x v)-t\right)\left(d_{G_{1}}(v y)-t\right) \\
& \left.+\sum_{x \in N_{G_{0}}(u) \cap N_{G_{0}}(v)}\left(d_{G_{1}}(u x)+t\right)\right)\left(d_{G_{1}}(x v)-t\right)+\frac{(r+t)(r+t-1)}{2}(q+a+r+t-1)^{2} .
\end{aligned}
$$


Then,

$$
\begin{aligned}
& E M_{2}\left(G_{2}\right)-E M_{2}\left(G_{1}\right)=-r \sum_{x \in N_{G_{0}}(u) \backslash N_{G_{0}}(v)} \sum_{y \in N_{G_{0}}(x) \backslash\{u\}} d_{G_{1}}(x y) \\
& -r \sum_{x, y \in N_{G_{0}}(u)}\left(d_{G_{1}}(x u)+d_{G_{1}}(u y)-r\right)+r \sum_{x, y \in N_{G_{0}}(v)}\left(d_{G_{1}}(x v)+d_{G_{1}}(v y)+r\right) \\
& -r(q+r+a-1) \sum_{x \in N_{G_{0}}(u)} d_{G_{1}}(x u)+r(q+r+2 t-1) \sum_{x \in N_{G_{0}}(v)} d_{G_{1}}(x v) \\
& +r \sum_{x \in N_{G_{0}}(v) \backslash N_{G_{0}}(u)} \sum_{y \in N_{G_{0}}(x) \backslash\{v\}} d_{G_{1}}(x y)-r \sum_{x \in N_{G_{0}}(u) \cap N_{G_{0}}(v)}\left[d_{G_{1}}(v x)-d_{G_{1}}(x u)\right] \\
& +r t(q+r+t-1)^{2}-r^{2}\left|N_{G_{0}}(u) \cap N_{G_{0}}(v)\right|+q r(r+t)(q+r+t-1) \\
& +\frac{1}{2} r(r-1)(t-a)(2 q+2 r+t+a-2) \frac{1}{2} r t(t-1)(2 q+r+2 t-2) . \\
& E M_{2}\left(G_{3}\right)-E M_{2}\left(G_{1}\right)=t \sum_{x \in N_{G_{0}}(u) \backslash N_{G_{0}}(v)} \sum_{y \in N_{G_{0}}(x) \backslash\{u\}} d_{G_{1}}(x y) \\
& +t \sum_{x, y \in N_{G_{0}}(u)}\left(d_{G_{1}}(x u)+d_{G_{1}}(u y)+t\right)-t \sum_{x, y \in N_{G_{0}}(v)}\left(d_{G_{1}}(x v)+d_{G_{1}}(v y)-t\right) \\
& +t(q+2 r+t+a-1) \sum_{x \in N_{G_{0}}(u)} d_{G_{1}}(x u)-t(q+t-1) \sum_{x \in N_{G_{0}}(v)} d_{G_{1}}(x v) \\
& \left.\sum_{x \in N_{G_{0}}(v) \backslash N_{G_{0}}(u)} d_{G_{1}}(x y)+t \sum_{y \in N_{G_{0}}(x) \backslash\{v\}} \sum_{x \in N_{G_{0}}(u) \cap N_{G_{0}}(v)}(v x)-d_{G_{1}}(x u)\right] \\
& +\frac{1}{2} r t(r-1)(2 q+2 r+t+2 a-2)+\frac{1}{2} t(t-1)(r+a)(2 q+r+2 t+a-2) . \\
& \sum_{G_{0}}(u) \cap N_{G_{0}}(v) \mid+t(q+a)(r+t)(q+r+t+a-1)+r t(q+r+t+a-1)^{2}
\end{aligned}
$$

Again, if one of the expressions (7) and (8) is greater than 0, then the lemma holds. Suppose $E M_{2}\left(G_{2}\right)-$ $E M_{2}\left(G_{1}\right) \leq 0$, then we will show that $E M_{2}\left(G_{3}\right)-E M_{2}\left(G_{1}\right)$ has to be greater than 0 . Similar to Subcase 1.1, we will first obtain a relation from Equation (7) by letting $E M_{2}\left(G_{2}\right)-E M_{2}\left(G_{1}\right) \leq 0$. That is, we get

$$
\begin{aligned}
& q(r+t)(q+r+t-1)+t(q+r+t-1)^{2}+\frac{1}{2}(r-1)(t-a)(2 q+2 r+t+a-2) \\
& +\frac{1}{2} t(t-1)(2 q+r+2 t-2)-r\left|N_{G_{0}}(u) \cap N_{G_{0}}(v)\right| \\
& \leq \sum_{x \in N_{G_{0}}(u) \backslash N_{G_{0}}(v)} \sum_{y \in N_{G_{0}}(x) \backslash\{u\}} d_{G_{1}}(x y)+\sum_{x, y \in N_{G_{0}}(u)}\left(d_{G_{1}}(x u)+d_{G_{1}}(u y)-r\right) \\
& +(q+r+a-1) \sum_{x \in N_{G_{0}}(u)} d_{G_{1}}(x u)-\sum_{x \in N_{G_{0}}(v) \backslash N_{G_{0}}(u)} \sum_{y \in N_{G_{0}}(x) \backslash\{v\}} d_{G_{1}}(x y) \\
& -\sum_{x, y \in N_{G_{0}}(v)}\left(d_{G_{1}}(x v)+d_{G_{1}}(v y)+r\right)-(q+r+2 t-1) \sum_{x \in N_{G_{0}}(v)} d_{G_{1}}(x v) \\
& +\sum_{x \in N_{G_{0}}(u) \cap N_{G_{0}}(v)}\left(d_{G_{1}}(v x)-d_{G_{1}}(x u)\right)
\end{aligned}
$$




$$
\begin{aligned}
& \leq \sum_{x \in N_{G_{0}}(u) \backslash N_{G_{0}}(v)} \sum_{y \in N_{G_{0}}(x) \backslash\{u\}} d_{G_{1}}(x y)+\sum_{x, y \in N_{G_{0}}(u)}\left(d_{G_{1}}(x u)+d_{G_{1}}(u y)+t\right) \\
& +(q+2 r+t+a-1) \sum_{x \in N_{G_{0}}(u)} d_{G_{1}}(x u)-\sum_{x \in N_{G_{0}}(v) \backslash N_{G_{0}}(u)} \sum_{y \in N_{G_{0}}(x) \backslash\{v\}} d_{G_{1}}(x y) \\
& -\sum_{x, y \in N_{G_{0}}(v)}\left[d_{G_{1}}(x v)+d_{G_{1}}(v y)-t\right]-(q+t-1) \sum_{x \in N_{G_{0}}(v)} d_{G_{1}}(x v) \\
& +\sum_{x \in N_{G_{0}}(u) \cap N_{G_{0}}(v)}\left[d_{G_{1}}(v x)-d_{G_{1}}(x u)\right] .
\end{aligned}
$$

Substituting for the first seven terms of right hand side of Equation (8) by the inequality (9), we have

$$
\begin{aligned}
& E M_{2}\left(G_{3}\right)-E M_{2}\left(G_{1}\right) \geq t\left\{q(r+t)(q+r+t-1)+t(q+r+t-1)^{2}\right. \\
& +r(q+r+t+a-1)^{2}+\frac{1}{2}(r-1)(t-a)(2 q+2 r+t+a-2)+\frac{1}{2} t(t-1)(2 q+r+2 t-2) \\
& +(q+a)(r+t)(q+r+t+a-1)+\frac{1}{2} r(r-1)(2 q+2 r+t+2 a-2) \\
& \left.+\frac{1}{2}(t-1)(r+a)(2 q+r+2 t+a-2)-(r+t)\left|N_{G_{0}}(u) \cap N_{G_{0}}(v)\right|\right\} \\
& >0, \text { since }\left|N_{G_{0}}(u) \cap N_{G_{0}}(v)\right| \leq q, \quad q \geq 2, \text { and } r, t \geq 1 .
\end{aligned}
$$

Case 2: If $u$ and $v$ are adjacent, then from the definition of $E M_{2}(G)$, we have

$$
\begin{aligned}
& E M_{2}\left(G_{1}\right)=\sum_{e_{i}, e_{j} \in E\left(G_{0} \backslash\{u, v\}\right)} d_{G_{1}}\left(e_{i}\right) d_{G_{1}}\left(e_{j}\right)+\sum_{x \in N_{G_{0}}(u) \backslash\left\{v, N_{G_{0}}(v)\right\}} \sum_{y \in N_{G_{0}}(x) \backslash\{u\}} d_{G_{1}}(u x) d_{G_{1}}(x y) \\
& +\sum_{x, y \in N_{G_{0}}(u) \backslash\{v\}} d_{G_{1}}(x u) d_{G_{1}}(u y)+\sum_{x \in N_{G_{0}}(v) \backslash\left\{u, N_{G_{0}}(u)\right\}} \sum_{y \in N_{G_{0}}(x) \backslash\{v\}} d_{G_{1}}(v x) d_{G_{1}}(x y) \\
& +\sum_{x, y \in N_{G_{0}}(v) \backslash\{u\}} d_{G_{1}}(x v) d_{G_{1}}(v y)+r(q+a+r-1) \sum_{x \in N_{G_{0}}(u) \backslash\{v\}} d_{G_{1}}(x u) \\
& +t(q+t-1) \sum_{x \in N_{G_{0}}(v) \backslash\{u\}} d_{G_{1}}(x v)+\sum_{x \in N_{G_{0}}(u) \cap N_{G_{0}}(v)} d_{G_{1}}(u x) d_{G_{1}}(x v) \\
& +\sum_{x \in N_{G_{0}}(u) \backslash\{v\}} d_{G_{1}}(x u) d_{G_{1}}(u v)+\sum_{x \in N_{G_{0}}(v) \backslash\{u\}} d_{G_{1}}(x v) d_{G_{1}}(v u) \\
& +t(q+t-1) d_{G_{1}}(u v)+r(r+q+a-1) d_{G_{1}}(u v)+\frac{r(r-1)}{2}(q+a+r-1)^{2} \\
& +\frac{t(t-1)}{2}(q+t-1)^{2} \\
& +\sum_{x, y \in N_{G_{0}}(u) \backslash\{v\}}\left(d_{G_{1}}(x u)-r\right)\left(d_{G_{1}}(u y)-r\right)+\sum_{x \in N_{G_{0}}(v) \backslash\left\{u, N_{G_{0}}(u)\right\}} \sum_{y \in N_{G_{0}}(x) \backslash\{v\}}\left(d_{G_{1}}(v x)+r\right) d_{G_{1}}(x y) \\
& +\sum_{x, y \in N_{G_{0}}(v) \backslash\{u\}}\left(d_{G_{1}}(x v)+r\right)\left(d_{G_{1}}(v y)+r\right)+(r+t)(q+r+t-1) \sum_{x \in N_{G_{0}}(v) \backslash\{u\}}\left(d_{G_{1}}(x v)+r\right) \\
& +\sum_{x \in N_{G_{0}}(u) \backslash\{v\}}\left(d_{G_{1}}(x u)-r\right) d_{G_{1}}(u v)+\sum_{x \in N_{G_{0}}(v) \backslash\{u\}}\left(d_{G_{1}}(x v)+r\right) d_{G_{1}}(u v) \\
& +\sum_{x \in N_{G_{0}}(u) \cap N_{G_{0}}(v)}\left(d_{G_{1}}(u x)-r\right)\left(d_{G_{1}}(x v)+r\right)+\frac{(r+t)((r+t-1))}{2}(q+r+t-1)^{2} \\
& +(r+t)(q+r+t-1) d_{G_{1}}(u v),
\end{aligned}
$$


and

$$
\begin{aligned}
& E M_{2}\left(G_{3}\right)=\sum_{e_{i}, e_{j} \in E\left(G_{0} \backslash\{u, v\}\right)} d_{G_{1}}\left(e_{i}\right) d_{G_{1}}\left(e_{j}\right)+\sum_{x \in N_{G_{0}}(u) \backslash\left\{v, N_{G_{0}}(v)\right\}} \sum_{y \in N_{G_{0}}(x) \backslash\{u\}}\left(d_{G_{1}}(u x)+t\right) d_{G_{1}}(x y) \\
& +\sum_{x, y \in N_{G_{0}}(u) \backslash\{v\}}\left(d_{G_{1}}(x u)+t\right)\left(d_{G_{1}}(u y)+t\right)+\sum_{x, y \in N_{G_{0}}(v) \backslash\{u\}}\left(d_{G_{1}}(x v)-t\right)\left(d_{G_{1}}(v y)-t\right) \\
& +\sum_{x \in N_{G_{0}}(v) \backslash\left\{u, N_{G_{0}}(u)\right\}} \sum_{y \in N_{G_{0}}(x) \backslash\{v\}}\left(d_{G_{1}}(v x)-t\right) d_{G_{1}}(x y)+\sum_{x \in N_{G_{0}}(u) \backslash\{v\}}\left(d_{G_{1}}(x u)+t\right) d_{G_{1}}(u v) \\
& +(r+t)(q+a+r+t-1) \sum_{x \in N_{G_{0}}(u) \backslash\{v\}}\left(d_{G_{1}}(x u)+t\right)+\sum_{x \in N_{G_{0}}(v) \backslash\{u\}}\left(d_{G_{1}}(x v)-t\right) d_{G_{1}}(u v) \\
& \left.+\sum_{x \in N_{G_{0}}(u) \cap N_{G_{0}}(v)}\left(d_{G_{1}}(u x)+t\right)\right)\left(d_{G_{1}}(x v)-t\right)+\frac{(r+t)((r+t-1))}{2}(q+a+r+t-1)^{2} \\
& +(r+t)(p+r+t-1) d_{G_{1}}(u v) .
\end{aligned}
$$

Then,

$$
\begin{aligned}
& E M_{2}\left(G_{2}\right)-E M_{2}\left(G_{1}\right)=-r \sum_{x \in N_{G_{0}}(u) \backslash\left\{v, N_{G_{0}}(v)\right\}} \sum_{y \in N_{G_{0}}(x) \backslash\{u\}} d_{G_{1}}(x y) \\
& -r \sum_{x, y \in N_{G_{0}}(u) \backslash\{v\}}\left[d_{G_{1}}(x u)+d_{G_{1}}(u y)-r\right]-r(q+r+a-1) \\
& +r \sum_{x \in N_{G_{0}}(v) \backslash\left\{u, N_{G_{0}}(u)\right\}} \sum_{y \in N_{G_{0}}(x) \backslash\{v\}} d_{G_{1}}(x y)+r \sum_{x, y \in N_{G_{0}}(u) \backslash\{v\}} d_{G_{1}}(x u) \backslash\{u\} \\
& \left.+r t(q+r+t-1)^{2}+d_{G_{1}}(v y)+r\right)+r(q+r+2 t-1) \sum_{x \in N_{G_{0}}(v) \backslash\{u\}} d_{G_{1}}(x v) \\
& -r \sum_{x \in N_{G_{0}}(u) \cap N_{G_{0}}(v)}\left(d_{G_{1}}(v x)-d_{G_{1}}(x u)\right)-r^{2}\left|N_{G_{0}}(u) \cap N_{G_{0}}(v)\right|+2 r(t-a) d_{G_{1}}(u v) \\
& +r(q-1)(r+t)(q+r+t-1)+\frac{r(r-1)}{2}(t-a)(2 q+2 r+t+a-1) \\
& +\frac{r t(t-1)}{2}(2 q+r+2 t-2)
\end{aligned}
$$

and

$$
\begin{aligned}
& E M_{2}\left(G_{3}\right)-E M_{2}\left(G_{1}\right)=t \sum_{x \in N_{G_{0}}(u) \backslash\left\{v, N_{G_{0}}(v)\right\}} \sum_{y \in N_{G_{0}}(x) \backslash\{u\}} d_{G_{1}}(x y) \\
& +t \sum_{x, y \in N_{G_{0}}(u) \backslash\{v\}}\left(d_{G_{1}}(x u)+d_{G_{1}}(u y)+t\right)+t(q+2 r+t+a-1) \sum_{x \in N_{G_{0}}(u) \backslash\{v\}} d_{G_{1}}(x u) \\
& -t \sum_{x \in N_{G_{0}}} \sum_{-t(v) \backslash\left\{u, N_{G_{0}}(u)\right\}} \sum_{y \in N_{G_{0}}(x) \backslash\{v\}} d_{G_{1}}(x y)-t \sum_{x, y \in N_{G_{0}}(v) \backslash\{u\}}\left(d_{G_{1}}(x v)+d_{G_{1}}(v y)-t\right) \\
& -t(q-1) \sum_{x \in N_{G_{0}}(v) \backslash\{u\}} d_{G_{1}}(x v)+t \sum_{x \in N_{G_{0}}(u) \cap N_{G_{0}}(v)}\left(d_{G_{1}}(v x)-d_{G_{1}}(x u)\right) \\
& +r t(q+r+t+a-1)^{2}+\frac{r t(r-1)}{2}(2 q+2 r+t+2 a-2) \\
& +\frac{t(t-1)}{2}(r+a)(2 q+r+2 t+a-2) .
\end{aligned}
$$

If either of the expressions in (13) or (14) is greater than 0, then the lemma holds. Suppose $E M_{2}\left(G_{2}\right)-$ $E M_{2}\left(G_{1}\right) \leq 0$, then we will show that $E M_{2}\left(G_{3}\right)-E M_{2}\left(G_{1}\right)$ has to be greater than 0 . Letting 
$E M_{2}\left(G_{2}\right)-E M_{2}\left(G_{1}\right) \leq 0$ in Equation (13), we have

$$
\begin{aligned}
& (q-1)(r+t)(q+r+t-1)+\frac{t(t-1)}{2}(2 q+r+2 t-2)+2(t-a) d_{G_{1}}(u v)+t(q+r+t-1)^{2} \\
& +\frac{1}{2}(r-1)(t-a)(2 q+2 r+t+a-1)-r\left|N_{G_{0}}(u) \cap N_{G_{0}}(v)\right| \\
& \leq \sum_{x \in N_{G_{0}}(u) \backslash\left\{v, N_{G_{0}}(v)\right\}} \sum_{y \in N_{G_{0}}(x) \backslash\{u\}} d_{G_{1}}(x y)+\sum_{x, y \in N_{G_{0}}(u) \backslash\{v\}}\left(d_{G_{1}}(x u)+d_{G_{1}}(u y)-r\right) \\
& +(q+r+a-1) \sum_{x \in N_{G_{0}}(u) \backslash\{v\}} d_{G_{1}}(x u)-\sum_{x \in N_{G_{0}}(v) \backslash\left\{u, N_{G_{0}}(u)\right\}} \sum_{y \in N_{G_{0}}(x) \backslash\{v\}} d_{G_{1}}(x y) \\
& \text { - } \sum_{x, y \in N_{G_{0}}(v) \backslash\{u\}}\left(d_{G_{1}}(x v)+d_{G_{1}}(v y)+r\right)-(q+r+2 t-1) \sum_{x \in N_{G_{0}}(v) \backslash\{u\}} d_{G_{1}}(x v) \\
& +\sum_{x \in N_{G_{0}}(u) \cap N_{G_{0}}(v)}\left(d_{G_{1}}(v x)-d_{G_{1}}(x u)\right) \\
& \leq \sum_{x \in N_{G_{0}}(u) \backslash\left\{v, N_{G_{0}}(v)\right\}} \sum_{y \in N_{G_{0}}(x) \backslash\{u\}} d_{G_{1}}(x y)+\sum_{x, y \in N_{G_{0}}(u) \backslash\{v\}}\left(d_{G_{1}}(x u)+d_{G_{1}}(u y)+t\right) \\
& +(q+2 r+t+a-1) \sum_{x \in N_{G_{0}}(u) \backslash\{v\}} d_{G_{1}}(x u)-\sum_{x \in N_{G_{0}}(v) \backslash\left\{u, N_{G_{0}}(u)\right\}} \sum_{y \in N_{G_{0}}(x) \backslash\{v\}} d_{G_{1}}(x y) \\
& -\sum_{x, y \in N_{G_{0}}(v) \backslash\{u\}}\left(d_{G_{1}}(x v)+d_{G_{1}}(v y)-t\right)-(q+t-1) \sum_{x \in N_{G_{0}}(v) \backslash\{u\}} d_{G_{1}}(x v) \\
& +\sum_{x \in N_{G_{0}}(u) \cap N_{G_{0}}(v)}\left(d_{G_{1}}(v x)-d_{G_{1}}(x u)\right) .
\end{aligned}
$$

Now, substituting for the first seven terms of right hand side of equation (14) by the inequality (15), we have

$$
\begin{aligned}
& E M_{2}\left(G_{3}\right)-E M_{2}\left(G_{1}\right) \geq t\left\{2(t-a) d_{G_{1}}(u v)+(q-1)(r+t)(q+r+t-1)\right. \\
& +\frac{1}{2}(r-1)(t-a)(2 q+2 r+t+a-1)+\frac{t(t-1)}{2}(2 q+r+2 t-2) \\
& +(q+a-1)(r+t)(q+r+t+a-1)+2(r+a) d_{G_{1}}(u v)+r(q+r+t+a-1)^{2} \\
& +\frac{r(r-1)}{2}(2 q+2 r+t+2 a-2)+\frac{(t-1)}{2}(r+a)(2 q+r+2 t+a-2) \\
& \left.+t(q+r+t-1)^{2}-(r+t)\left|N_{G_{0}}(u) \cap N_{G_{0}}(v)\right|\right\} .
\end{aligned}
$$

Since $\left|N_{G_{0}}(u) \cap N_{G_{0}}(v)\right| \leq q$ and $d_{G_{1}}(u v)=(2 q+r+t+a-2)$, then above inequality can be written as

$$
\begin{aligned}
& E M_{2}\left(G_{3}\right)-E M_{2}\left(G_{1}\right) \geq t\{2(t+a)(2 q+r+t+a-2)+(q-1)(r+t)(q+r+t-1) \\
& +\frac{1}{2}(r-1)(t-a)(2 q+2 r+t+a-1)+\frac{t(t-1)}{2}(2 q+r+2 t-2)+r(q+r+t+a-1)^{2} \\
& +(q+a-1)(r+t)(q+r+t+a-1)+\frac{r(r-1)}{2}(2 q+2 r+t+2 a-2) \\
& \left.+\frac{(t-1)}{2}(r+a)(2 q+r+2 t+a-2)+t(q+r+t-1)^{2}-(r+t) q\right\} \\
& >0, \text { since } q \geq 2, \text { and } r, t \geq 1 .
\end{aligned}
$$

Remark 2.1 If $G_{0}=C_{k}$, where $C_{k}$ is the cycle graph on $k$ vertices, then in Lemma 2.3, vertices $u$ and $v$ have the same degree in graph $G_{0}$. 
Remark 2.2 If $G_{0}$ is a bicyclic graph which has no pendant vertex, see Figure 7. Then, in Lemma 2.3, possible degree of vertex $u$ and $v$ in graph $G_{0}$ are $p=q$ or $p=q+1$ or $p=q+2$.

To estimate the upper bounds of $E M_{2}(G)$ for trees, unicyclic, and bicyclic graphs we will use the transformations $\mathbf{A}, \mathbf{B}$ and $\mathbf{C}$.

\section{Three transformations which decrease the second reformulated Zagreb index}

In this section, we make use of three more graph operations as defined in (Deng, 2007; Ji et al., 2014) that are useful for finding the lower bounds of $E M_{2}(G)$.

Transformation D: Let $G_{0}$ be a non-trivial graph and $u$ be any vertex of $G_{0}$. Let $G_{1}$ be a graph obtained from $G_{0}$ by attaching at $u$ two paths $P_{1}=u u_{1} u_{2} \ldots u_{r}$ of length $r$ and $P_{2}=u v_{1} v_{2} \ldots v_{t}$ of length $t$. Let $G_{2}=G_{1}-\left\{u u_{1}\right\}+\left\{v_{t} u_{1}\right\}$; see Figure 4 . Here $G_{2}$ is said to be obtained from $G_{1}$ by transformation D.

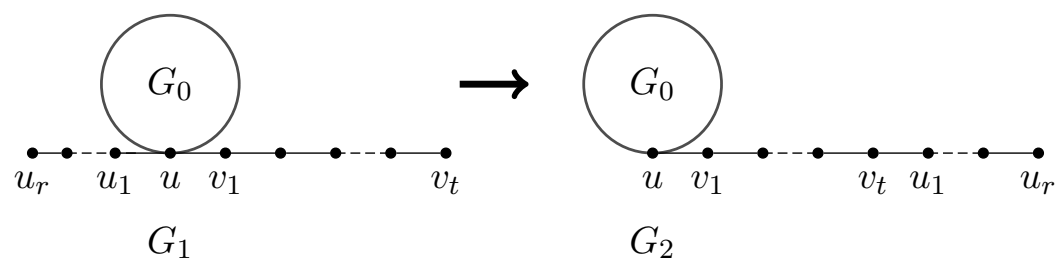

Fig. 4. Representation of transformation $D$

Lemma 3.1 Let $G_{2}$ be a graph obtained by Transformation $D$ from $G_{1}$ as shown in Figure 4. Then, $E M_{2}\left(G_{1}\right)>E M_{2}\left(G_{2}\right)$.

Proof: Let $N_{G_{0}}(u)$ denote the neighborhood of the vertex of $u$ in $G_{0}$. Now, we prove this lemma by taking condition on path length $r, t$.

If $r, t \geq 3$ then by definition of $E M_{2}(G)$, we have

$$
\begin{aligned}
& E M_{2}\left(G_{1}\right)-E M_{2}\left(G_{2}\right)=\sum_{x \in N_{G_{0}}(u)} \sum_{y \in N_{G_{0}}(x) \backslash\{u\}} d_{G_{1}}(x y)+\sum_{x, y \in N_{G_{0}}(u)}\left(d_{G_{1}}(x u)+d_{G_{1}}(u y)-1\right) \\
& +\sum_{x \in N_{G_{0}}(u)}\left(\left(d_{G_{1}}(u)+1\right) d_{G_{1}}(u x)+\left(d_{G_{1}}(u)-1\right)\right)+d_{G_{1}}^{2}(u)+2 d_{G_{1}}(u)-8 \\
& >0, \text { since } d_{G_{1}}(u)>2 .
\end{aligned}
$$

Similarly proof holds for the remaining values of $r$ and $t$, that is, for $r=2, t \geq 3 ; r=1, t \geq 3$; $r=2, t=2 ; r=1, t=2$ and $r=1, t=1$. We omit the proofs for brevity.

Remark 3.1 Note that, we can convert any tree into a path graph by repeating transformation $D$.

Transformation E: Let $G_{0}$ be a non-trivial graph having vertices $u$ and $v$. Let $G_{1}$ be a graph obtain from $G_{0}$ by adding two paths $\left\langle u, u_{1}, u_{2}, \ldots, u_{r}>\right.$ and $\left\langle v, v_{1}, v_{2}, \ldots, v_{t}\right\rangle$ at vertices $u$ and $v$ of length $r$ and $t$, respectively. Let $G_{2}:=G_{1}-\left\{u u_{1}\right\}+\left\{v_{t} u_{1}\right\}$. Here, $G_{2}$ is said to be obtained from $G_{1}$ by transformation E; see Figure 5.

Lemma 3.2 Let $G_{2}$ be the graph obtained from $G_{1}$ by using transformation $E$, then $E M_{2}\left(G_{1}\right)>E M_{2}\left(G_{2}\right)$.

Proof: We prove this lemma by making conditions on the path lengths $r$ and $t$. First, we assume that $r \geq 3$ and $t \geq 3$. Now we have the following cases. 


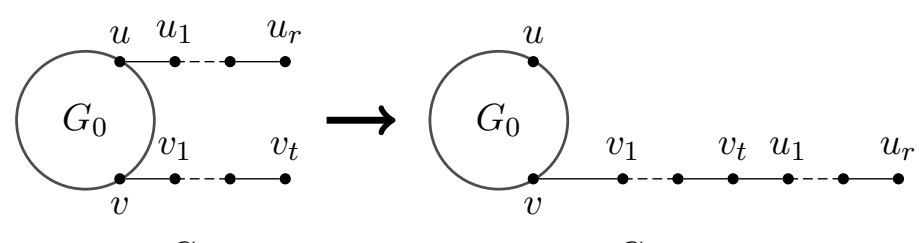

$G_{1}$

$G_{2}$

Fig. 5. Representation of transformation E.

Case 1: If vertex $u$ and $v$ are not adjacent, then we have two subcases.

Subcase 1.1: If $N_{G_{0}}(u) \cap N_{G_{0}}(v)=\emptyset$, then from definition of $E M_{2}(G)$, we have

$$
\begin{aligned}
E M_{2}\left(G_{1}\right)-E M_{2}\left(G_{2}\right)= & \sum_{x \in N_{G_{0}}(u)} \sum_{y \in N_{G_{0}}(x) \backslash\{u\}} d_{G_{1}}(x y)+\sum_{x, y \in N_{G_{0}}(u)}\left(d_{G_{1}}(x u)+d_{G_{1}}(u y)-1\right) \\
& +\sum_{x \in N_{G_{0}}(u)} d_{G_{1}}(x u) d_{G_{1}}\left(u u_{1}\right)+2 d_{G_{1}}\left(u u_{1}\right)-10 \\
& \geq d_{G_{1}}(x u) d_{G_{1}}\left(u u_{1}\right)+2 d_{G_{1}}\left(u u_{1}\right)-10 \\
& >0, \text { since } d_{G_{1}}(x u) \geq 2, d_{G_{1}}\left(u u_{1}\right) \geq 3 .
\end{aligned}
$$

Subcase 1.2: If $N_{G_{0}}(u) \cap N_{G_{0}}(v) \neq \emptyset$, then from definition of $E M_{2}(G)$, we have

$$
\begin{aligned}
E M_{2}\left(G_{1}\right)-E M_{2}\left(G_{2}\right) & =\sum_{x \in N_{G_{0}}(u) \backslash N_{G_{0}}(v)} \sum_{y \in N_{G_{0}}(x) \backslash\{u\}} d_{G_{1}}(x y)+\sum_{x, y \in N_{G_{0}}(u)}\left(d_{G_{1}}(x u)+d_{G_{1}}(u y)-1\right) \\
& +\sum_{x \in N_{G_{0}}(u)} d_{G_{1}}(x u) d_{G_{1}}\left(u u_{1}\right)+\sum_{x \in N_{G_{0}}(u) \cap N_{G_{0}}(v)} d_{G_{1}}(x v)+2 d_{G_{1}}\left(u u_{1}\right)-10 \\
& \geq d_{G_{1}}(x u) d_{G_{1}}\left(u u_{1}\right)+2 d_{G_{1}}\left(u u_{1}\right)-10 \\
& >0, \text { since } d_{G_{1}}(x u) \geq 2, d_{G_{1}}\left(u u_{1}\right) \geq 3 .
\end{aligned}
$$

Case 2 If vertex $u$ and $v$ are adjacent then from definition of $E M_{2}(G)$, we have

$$
\begin{aligned}
& E M_{2}\left(G_{1}\right)-E M_{2}\left(G_{2}\right)=\sum_{x \in N_{G_{0}}(u) \backslash\left\{v, N_{G_{0}}(v)\right\}} \sum_{y \in N_{G_{0}}(x) \backslash\{u\}} d_{G_{1}}(x y)+\sum_{x \in N_{G_{0}}(u) \cap N_{G_{0}}(v)} d_{G_{1}}(x v) \\
& +\sum_{x, y \in N_{G_{0}}(u) \backslash\{v\}}\left(d_{G_{1}}(x u)+d_{G_{1}}(u y)-1\right)+\sum_{x \in N_{G_{0}}(u) \backslash\{v\}}\left(d_{G_{1}}(x u)+d_{G_{1}}(u v)-1\right) \\
& +\sum_{x \in N_{G_{0}}(v) \backslash\{u\}} d_{G_{1}}(x v)+d_{G_{1}}\left(v v_{1}\right)+2 d_{G_{1}}\left(u u_{1}\right)+d_{G_{1}}\left(u u_{1}\right) d_{G_{1}}(u v)-10 \\
& \geq d_{G_{1}}\left(v v_{1}\right)+2 d_{G_{1}}\left(u u_{1}\right)+d_{G_{1}}\left(u u_{1}\right) d_{G_{1}}(u v)-10 \\
& >0, \text { since } d_{G_{1}}\left(v v_{1}\right) \geq 3, d_{G_{1}}\left(u u_{1}\right) \geq 3 \text { and } d_{G_{1}}(u v) \geq 3 .
\end{aligned}
$$

Proof is complete for $r, t \geq 3$.

Similarly proof holds for the remaining values of $r$ and $t$, that is, for $(r, t)$ is one of the pairs $(1,2)$, $(1,3),(2,2)$ and $(2,3)$. We omit the proofs for brevity.

Remark 3.2 By suitable and repeated application of transformations $D$ and $E$ to any unicyclic graph on $n$ vertices and having girth $k$, we can obtain a $(k, n-k)$-tadpole graph. We denote $a(k, n-k)$-tadpole graph by $C_{n}^{k}$.

Note: A $(p, q)$-tadpole graph is a special type of unicyclic graph obtained by attaching a path graph on $q$ vertices to a cycle graph on $p$ (at least 3 ) vertices with a bridge. 
Transformation F: Let $G_{0}$ be a graph containing a path of length at least 2 , say $\left\langle u, x_{1}, v\right\rangle$. Let $G_{1}$ be the graph obtained from $G_{0}$ by attaching a path $\left\langle x_{1}, x_{2}, \ldots, x_{k}>,(k \geq 1)\right.$ at $x_{1}$. Let $G_{2}:=$ $G_{1}-\left\{x_{1} v\right\}+\left\{x_{k} v\right\}$; see Figure 6 . We say that $G_{2}$ is obtained from $G_{1}$ by transformation $\mathbf{F}$.

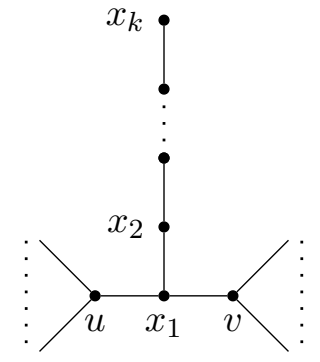

$G_{1}$

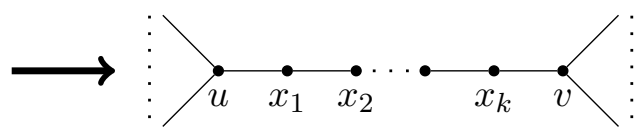

$G_{2}$

Fig. 6. Representation of transformation F

Lemma 3.3 If $G_{2}$ is obtained from $G_{1}$ using transformation $F$, then $E M_{2}\left(G_{1}\right)>E M_{2}\left(G_{2}\right)$.

Proof: From the definition of $E M_{2}(G)$ and using the fact that $d_{G_{2}}\left(u x_{1}\right)=d_{G_{1}}\left(u x_{1}\right)-1, d_{G_{2}}\left(x_{k} v\right)=$ $d_{G_{1}}\left(x_{1} v\right)-1$, we have

$$
\begin{aligned}
E M_{2}\left(G_{1}\right)-E M_{2}\left(G_{2}\right) & \geq d_{G_{1}}\left(u x_{1}\right) d_{G_{1}}\left(x_{1} v\right)+2 d_{G_{1}}\left(u x_{1}\right)+2 d_{G_{1}}\left(x_{1} v\right)-2 d_{G_{2}}\left(u x_{1}\right)-2 d_{G_{2}}\left(x_{k} v\right) \\
& =d_{G_{1}}\left(u x_{1}\right) d_{G_{1}}\left(x_{1} v\right)+4>0 .
\end{aligned}
$$

Remark 3.3 Similarly, repeated application of the transformations $D$ and $E$ to a bicyclic graph will result in such a bicyclic graph where a path is attached to one of the graphs in Figure 7 and finally applying transformation $F$ to the resultant graph will result in exactly one of the graphs in Figure 7.

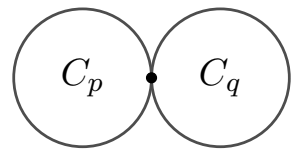

(a) $D_{n}^{1}(p, q)$

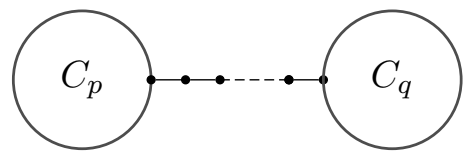

(b) $D_{n}^{2}(p, q, l)$

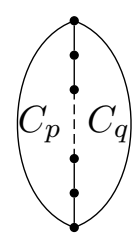

(c) $D_{n}^{3}(p, q, l)$

Fig. 7. Bicyclic graphs which has no pendant vertices

\section{Bounds of second reformulated Zagreb index}

In this section, we compute the upper and lower bounds for trees, unicyclic, bicyclic and tricyclic graphs using the six transformations discussed in sections 2 and 3.

\subsection{Trees}

It is well known that the path graph is the only tree on 3 vertices and $E M_{2}\left(P_{3}\right)=1$. For trees on at least 4 vertices we have the following theorem.

Theorem 4.1 If $T \neq\left\{P_{n}, S_{n}\right\}$ is a tree on $n \geq 4$ vertices, then $4 n-12=E M_{2}\left(P_{n}\right)<E M_{2}(T)<$ $E M_{2}\left(S_{n}\right)=\frac{1}{2}(n-1)(n-2)^{3}$.

Proof: Since any tree $T$ can be transformed into a star $S_{n}$ by using the tranformations $A$ and $C$, it follows immediately from Lemma 2.1 and Lemma 2.3, $E M_{2}\left(S_{n}\right)>E M_{2}(T)$.

Similarly, for the lower bound, we can see that the result follows directly from Lemma 3.1 and Remark 
3.1.

By direct computation using the definition of the second reformulated Zagreb index, it follows that $E M_{2}\left(P_{n}\right)=4 n-12$ and $E M_{2}\left(S_{n}\right)=\frac{1}{2}(n-1)(n-2)^{3}$ and hence the theorem holds.

From the above theorem, it is immediate that among the trees on $n$ vertices, the path graph attains the least value and the star graph attains the greatest value for the second reformulated Zagreb index.

\subsection{Unicyclic Graphs}

Before finding the upper bounds for the unicyclic graph, let us fix some notation. Let $U_{n}^{k}$ denotes the unicyclic graph obtained from the cycle $C_{k}$ of girth $k$ by attaching $n-k$ pendent vertices to any one vertex on $C_{k}$. Let $C_{n}^{k}$ denotes the tadpole graph with girth $k$, that is, the cycle of girth $k$ is connected to a path $P_{n-k}$ with a bridge.

Theorem 4.2 If $G$ is a unicyclic graph on $n$ vertices and girth $k$, then $E M_{2}(G) \leq E M_{2}\left(U_{n}^{k}\right)$. Equality holds if and only if $G \simeq U_{n}^{k}$

Proof: Proof follows from Lemma 2.1 and Lemma 2.3 as any unicyclic graph can be transformed into $U_{n}^{k}$ by using transformations $\mathrm{A}$ and $\mathrm{C}$.

Corollary 4.1 For fixed $n \geq 4$ and girth $4 \leq k \leq n, E M_{2}\left(U_{n}^{k}\right) \leq E M_{2}\left(U_{n}^{k-1}\right)$.

Proof: From the definition of $E M_{2}(G)$, we have upon simplification

$$
E M_{2}\left(U_{n}^{k}\right)=\frac{1}{2}(n-k+1)^{2}\left[(n-k)^{2}+3(n-k+2)\right]+(4 n-3)
$$

Therefore,

$$
\begin{aligned}
E M_{2}\left(U_{n}^{k}\right)-E M_{2}\left(U_{n}^{k-1}\right) & =-\frac{1}{2}(n-k)(n-k+1)(4 n-4 k+5) \\
& -6(n-k+2)(n-k+1)-2(n-k+2)-1 \\
& \leq 0
\end{aligned}
$$

Hence the proof.

Theorem 4.3 $U_{n}^{3}$ is the unique graph with the largest $E M_{2}(G)$ among all the unicyclic graphs.

Proof: From Theorem 4.2 and Corollary 4.1, the result follows immediately. The value of $E M_{2}\left(U_{n}^{3}\right)$ can be directly computed and is given by $E M_{2}\left(U_{n}^{3}\right)=\frac{1}{2}(n-3)(n-4)(n-2)^{2}+(n-1)\left(2 n^{2}-9 n+15\right)$.

Theorem 4.4 Let $G$ be a unicyclic graph on $n$ vertices. If $G \neq\left\{C_{n}, C_{n}^{k}\right\}$, then $E M_{2}(G)>E M_{2}\left(C_{n}^{k}\right)>$ $E M_{2}\left(C_{n}\right)$, where $C_{n}^{k}$ is a tadpole graph.

Proof: From Remark 3.2, Lemma 3.1, Lemma 3.2, and Lemma 3.3, the result follows immediately and we have $E M_{2}(G)>E M_{2}\left(C_{n}^{k}\right)>E M_{2}\left(C_{n}\right)$.

Theorem 4.5 The value of $E M_{2}\left(C_{n}\right)=4 n$ and $E M_{2}\left(C_{n}^{k}\right)= \begin{cases}4 n+17, & n=k+1 \\ 4 n+22, & n=k+2 \\ 4 n+23, & n \geq k+3\end{cases}$

Proof: Proof follows by direct computation. 


\subsection{Bicyclic Graphs}

Before proving the bounds of bicyclic graph, we define some notations. Let $\mathbb{B}(n, n+1)$ be the set of bicyclic graphs of $n$ vertices and $n+1$ edges. Any bicyclic graph belong to any one of the following collection of bicyclic graphs.

1. Let $\mathbb{B}_{n}^{1}(p, q) \subset \mathbb{B}(n, n+1)$ be the set of bicyclic graphs on $n$ vertices, where $p+q-1 \leq n$, is such that if $G \in \mathbb{B}_{n}^{1}(p, q)$, then two cycles $C_{p}$ and $C_{q}$ are attached by a common vertex in $G$. Let $D_{n}^{1}(p, q) \subset \mathbb{B}_{n}^{1}(p, q)$ be the set of graphs having exactly $n=p+q-1$ vertices, that is, if $G \in D_{n}^{1}(p, q)$, then $G$ has no any pendant vertex, see Figure 7(a).

2. Let $\mathbb{B}_{n}^{2}(p, q, l) \subset \mathbb{B}(n, n+1)$ be the set of bicyclic graphs on $n$ vertices, where $p+q+l \leq n+1$, is such that if $G \in \mathbb{B}_{n}^{2}(p, q, l)$, then the two cycles $C_{p}$ and $C_{q}$ are connected by a path of length $l \geq 1$ in $G$. Let $D_{n}^{2}(p, q, l) \subset \mathbb{B}_{n}^{2}(p, q, l)$ be the set of graphs having exactly $n+1=p+q+l$ vertices, that is, if $G \in D_{n}^{2}(p, q, l)$, then $G$ has no pendant vertex, see Figure 7(b).

3. Let $\mathbb{B}_{n}^{3}(p, q, l) \subset \mathbb{B}(n, n+1)$ be the set of bicyclic graphs on $n$ vertices, where $p+q-l \leq n+1$, is such that if $G \in \mathbb{B}_{n}^{3}(p, q, l)$, then the two cycles $C_{p}$ and $C_{q}$ have a common path of length $l \geq 1$ in $G$. Let $D_{n}^{3}(p, q, l) \subset \mathbb{B}_{n}^{3}(p, q, l)$ be the set of graphs having exactly $n+1=p+q-l$ vertices, that is, if $G \in D_{n}^{3}(p, q, l)$, then $G$ has no pendant vertex, see Figure 7(c).

Theorem 4.6 $F_{n}^{3}$ is the unique graph which has the largest value of $E M_{2}(G)$ among all bicyclic graph.

Proof:Let $G \in \mathbb{B}(n, n+1)$ be a bicyclic graph. $G$ lies in one of the classes of bicyclic graphs $\mathbb{B}_{n}^{1}(p, q)$, $\mathbb{B}_{n}^{2}(p, q, l), \mathbb{B}_{n}^{3}(p, q, l)$.

Suppose $G \in \mathbb{B}_{n}^{1}(p, q)$ or $G \in \mathbb{B}_{n}^{2}(p, q, l)$ : By repeated and suitable application of the operations $A$, $B$ and $C$ on graph $G, G$ is transformed into one of the graphs of the form $F_{n}^{1}$ or $F_{n}^{2}$ as shown in Figure $8\left(\right.$ a) or 8(b), respectively. Hence by Lemma 2.1, Lemma 2.2 and Lemma 2.3, we have $E M_{2}(G) \leq$ $E M_{2}\left(F_{n}^{1}\right)$ or $E M_{2}(G) \leq E M_{2}\left(F_{n}^{2}\right)$, respectively.

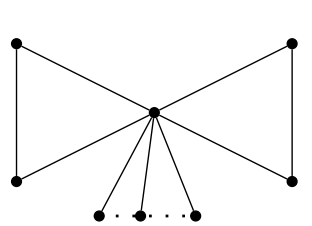

(a) $F_{n}^{1}$

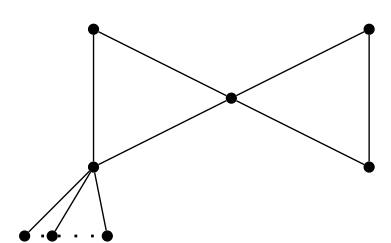

(b) $F_{n}^{2}$

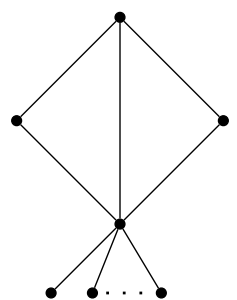

(c) $F_{n}^{3}$

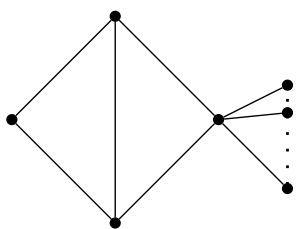

(d) $F_{n}^{4}$

Fig. 8. Some bicyclic graphs which are using in upper bounds

Similarly, if $G \in \mathbb{B}_{n}^{3}(p, q)$ and upon repeated application of the transformations $A, B$ and $C$ suitably we can convert the graph $G$ into one of the graphs of the form $F_{n}^{3}$ or $F_{n}^{4}$ as shown in Figure 8(c), 8(d), respectively. Hence by Lemma 2.1, Lemma 2.2 and Lemma 2.3, we have $E M_{2}(G) \leq E M_{2}\left(F_{n}^{3}\right)$ or $E M_{2}(G) \leq E M_{2}\left(F_{n}^{4}\right)$.

As a resultant, we only need to compare the values of $E M_{2}$ for graphs in $F_{n}^{1}, F_{n}^{2}, F_{n}^{3}$ and $F_{n}^{4}$. By direct computation, we obtain the following.

$$
\begin{array}{ll}
E M_{2}\left(F_{n}^{1}\right)=\frac{1}{2}\left\{n^{4}-7 n^{3}+26 n^{2}-36 n+36\right\}, & E M_{2}\left(F_{n}^{2}\right)=\frac{1}{2}\left\{n^{4}-15 n^{3}+92 n^{2}-240 n+406\right\} \\
E M_{2}\left(F_{n}^{3}\right)=\frac{1}{2}\left\{n^{4}-7 n^{3}+26 n^{2}-28 n+56\right\}, & E M_{2}\left(F_{n}^{4}\right)=\frac{1}{2}\left\{n^{4}-11 n^{3}+53 n^{2}-101 n+172\right\}
\end{array}
$$

Thus, by comparing the above values, we get

$$
E M_{2}(G) \leq \frac{1}{2}\left\{n^{4}-7 n^{3}+26 n^{2}-28 n+56\right\}=E M_{2}\left(F_{n}^{3}\right)
$$


Theorem 4.7 If $G$ is a bicyclic graph on $n \geq 8$ vertices, then $E M_{2}(G) \geq 4 n+58$. Equality holds if and only if either $G \in D_{n}^{2}(p, q, l)$ for $l \geq 3$ or $G \in D_{n}^{3}(p, q, l)$ for $p \geq 6, q \geq 6, l \geq 3$.

Proof: From our observation in Remark 3.3, it is immediate that any bicyclic graph $G$ on $n$ vertices can be transformed into a graph in one of the classes $D_{n}^{1}(p, q), D_{n}^{2}(p, q, l)$, or $D_{n}^{3}(p, q, l)$ on $n$ vertices using repeated application of transformations $D, E$ and $F$. Hence by Lemmas 3.2 and Lemma 3.3, the smallest value of $E M_{2}(G)$ is attained by the graphs in one of the above classes.

As a resultant, we only need to compare the value of $E M_{2}$ for graphs in each of the class $D_{n}^{1}(p, q)$, $D_{n}^{2}(p, q, l)$, and $D_{n}^{3}(p, q, l)$. As the computations are straight forward, we present the values directly.

For a graph $H \in D_{n}^{1}(p, q), E M_{2}(H)=4 n+108, n \geq 5$.

For $H \in D_{n}^{2}(p, q, l)$, the cycles $C_{p}$ and $C_{q}$ are connected by a path of length $l \geq 1$. By direct caluclation, we have $E M_{2}(H)=4 n+66, n \geq 6$ and $H \in D_{n}^{2}(p, q, 1)$.

$E M_{2}(H)=4 n+59, n \geq 7$ and $H \in D_{n}^{2}(p, q, 2)$.

$E M_{2}(H)=4 n+58, n \geq 8$ and $H \in D_{n}^{2}(p, q, l)$ for $l \geq 3$.

For $H \in D_{n}^{3}(p, q, l)$, recall in $H$, the cycles $C_{p}$ and $C_{q}$ have a common path of length $l \geq 1$.

1. If $n=4$ or $(p=3, q=3$ and $l=1)$, then $E M_{2}\left(D_{4}^{3}(3,3,1)\right)=84$.

2. If $H \in D_{n}^{3}(p, 3,1)$ for $n \geq 5$, and $p \geq 4$, then $E M_{2}(H)=4 n+67$.

3. If $H \in D_{n}^{3}(p, q, 1)$ for $n \geq 6, p \geq 4$, and $q \geq 4$, then $E M_{2}(H)=4 n+66$.

4. If $n=5$ or $(p=4, q=4$ and $l=2)$, then $E M_{2}\left(D_{3}(4,4,2)\right)=81$.

5. If $H \in D_{n}^{3}(p, 4,2)$ for $n \geq 6$ and $p \geq 5$, then $E M_{2}(H)=4 n+60$.

6. If $H \in D_{n}^{3}(p, q, 2)$ for $n \geq 7, p \geq 5$ and $q \geq 5$, then $E M_{2}(H)=4 n+59$.

7. If $H \in D_{n}^{3}(p, q, l)$ for $n \geq 8, p \geq 6, q \geq 6$ and $l \geq 3$, then $E M_{2}(H)=4 n+58$.

Thus, from the above values we get the desired results.

\subsection{Tricyclic Graphs}

Before proving the bounds of tricyclic graphs, we will fix some notations that will be used in our proof. Let $\mathbb{X}^{n}$ be the set of tricyclic graphs of $n$ vertices. We define two interesting sub collections of tricyclic graphs that are required.

1. Let $\mathbb{X}_{0}^{n} \subset \mathbb{X}^{n}$ be the set of tricyclic graphs of $n$ vertices such that if $G \in \mathbb{X}_{0}^{n}$, then $G$ has no pendent vertices. There are 15 possible collections of graphs in $\mathbb{X}_{0}^{n}$, whose representation is given in Figure 10.

2. Let $\mathbb{X}_{1}^{n} \subset \mathbb{X}_{0}^{n}$ be the set of tricyclic graphs such that if $G \in \mathbb{X}_{1}^{n}$, then $G$ has maximum vertex degree three and any two vertices of degree three are connected by a path of length atleast two in $G$. Representation of graphs in $\mathbb{X}_{1}^{n}$ is given in Figure 11.

Theorem 4.8 Let $G \in \mathbb{X}^{n}$ be a tricyclic graph of vertex $n$. Then

$$
E M_{2}(G) \leq \frac{1}{2}\left(n^{4}-7 n^{3}+30 n^{2}-20 n+176\right)
$$

where the equality holds if and only if $G \simeq \gamma_{5}^{n}$ or $G \simeq \gamma_{6}^{n}$, as in Figure 9 . 


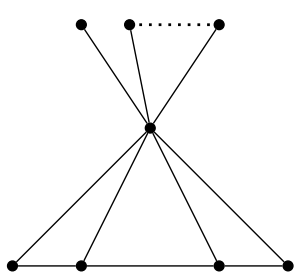

(a) $\gamma_{1}^{n}$

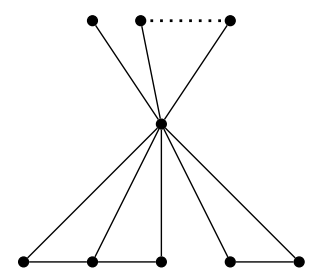

(b) $\gamma_{2}^{n}$

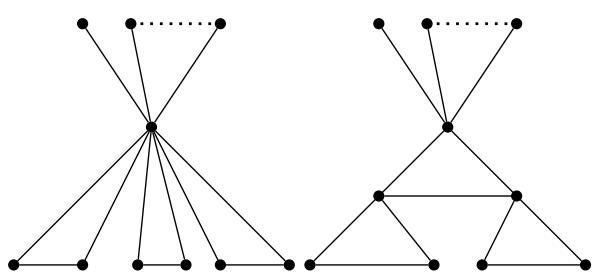

(c) $\gamma_{3}^{n}$

(d) $\gamma_{4}^{n}$

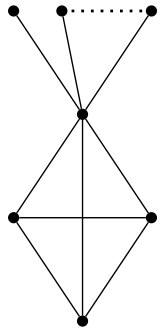

(f) $\gamma_{6}^{n}$

Fig. 9. Tricyclic graphs which attains the upper bounds

Proof: If, we are repeating transformations $A, B$ and $C$, then, the tricyclic graph $G$ can be converted into one of the six graphs shown in Figure 9. Then, from Lemma 2.1, Lemma 2.2 and Lemma 2.3, we have $E M_{2}(G) \leq E M_{2}\left(\gamma_{i}^{n}\right), i=1, \ldots, 6$. Hence, as before, we only need to compare the value of $E M_{2}\left(\gamma_{i}^{n}\right)$, $i=1, \ldots, 6$. By direct computation we have

$$
\begin{array}{ll}
E M_{2}\left(\gamma_{1}^{n}\right)=\frac{1}{2}\left(n^{4}-7 n^{3}+30 n^{2}-28 n+118\right), & E M_{2}\left(\gamma_{2}^{n}\right)=\frac{1}{2}\left(n^{4}-7 n^{3}+30 n^{2}-36 n+82\right) \\
E M_{2}\left(\gamma_{3}^{n}\right)=\frac{1}{2}\left(n^{4}-7 n^{3}+30 n^{2}-44 n+62\right), & E M_{2}\left(\gamma_{4}^{n}\right)=\frac{1}{2}\left(n^{4}-23 n^{3}+210 n^{2}-844 n+1682\right) \\
E M_{2}\left(\gamma_{5}^{n}\right)=\frac{1}{2}\left(n^{4}-7 n^{3}+30 n^{2}-20 n+176\right), & E M_{2}\left(\gamma_{6}^{n}\right)=\frac{1}{2}\left(n^{4}-7 n^{3}+30 n^{2}-20 n+176\right) .
\end{array}
$$

Thus, by comparing the above expressions, we get

$$
E M_{2}(G) \leq \frac{1}{2}\left(n^{4}-7 n^{3}+30 n^{2}-20 n+176\right) .
$$

Hence we see that $G$ attains the upper bound when $G$ is isomorphic to a graph in $\gamma_{5}^{n}$ or $\gamma_{6}^{n}$.

Theorem 4.9 Let $G \in \mathbb{X}^{n}$ be a tricyclic graph of vertex $n$. Then $E M_{2}(G) \geq 4 n+116$. Equality holds if and only if $G \in \mathbb{X}_{1}^{n}$.

Proof: By repeated application of the operations $D, E$ and $F$ to the tricyclic graph $G$, we can transform the graph $G$ into one of the 15 tricyclic graphs as shown in Figure 10. From Lemma 3.1, Lemma 3.2, and Lemma 3.3, we have $E M_{2}(G) \geq E M_{2}\left(\alpha_{i}^{n}\right)$, where $\alpha_{i}^{n} \in \mathbb{X}_{0}^{n}$, for $1 \leq i \leq 15$. Among the graphs in $\mathbb{X}_{0}^{n}$, we observe that the five collection of graphs in $\mathbb{X}_{1}^{n}$ attain the lowest value of $E M_{2}$. By direct computation, we get

$$
E M_{2}\left(\alpha_{i}\right) \geq E M_{2}\left(\beta_{j}^{n}\right)=4 n+116,
$$

where $\beta_{j}^{n} \in \mathbb{X}_{1}^{n} \subset \mathbb{X}_{0}^{n}, j=1, \ldots, 5$.

\section{Conclusion}

In this paper, we have computed the bounds of the second reformulated Zagreb index, $\operatorname{EM}_{2}(G)$, using six different graph transformations. In this process, we also identify the extremal graphs which attain that bound for trees, unicyclic, bicyclic and tricyclic graphs. Our focus here was to study the graphs with cyclomatic number at most 3 . In the future, we would like to extend this study to any graphs particularly planar graphs. 


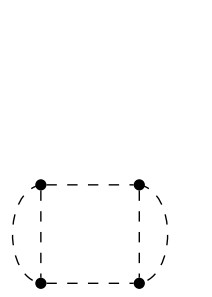

(a) $\alpha_{1}^{n}$

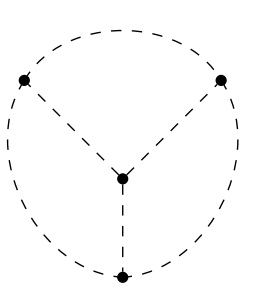

(b) $\alpha_{2}^{n}$ (c) $\alpha_{3}^{n}$

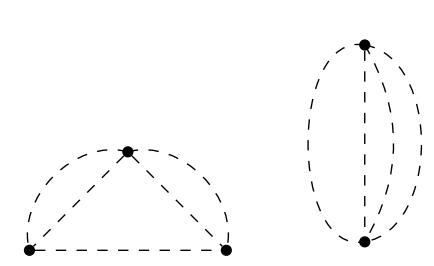

(d) $\alpha_{4}^{n}$

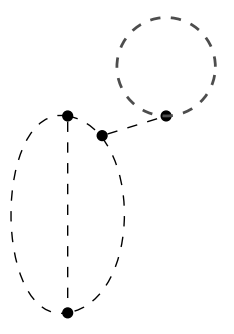

(e) $\alpha_{5}^{n}$

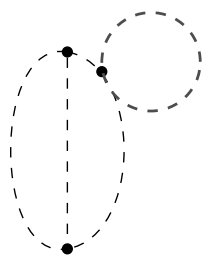

(f) $\alpha_{6}^{n}$

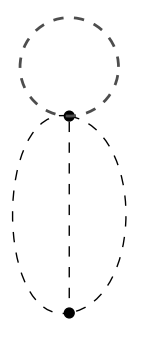

(g) $\alpha_{7}^{n}$

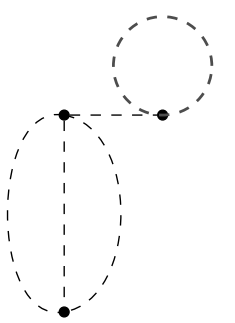

(h) $\alpha_{8}^{n}$

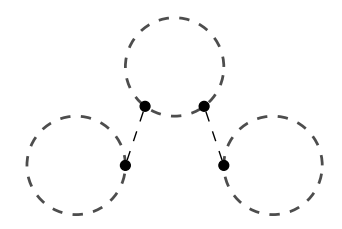

(i) $\alpha_{9}^{n}$

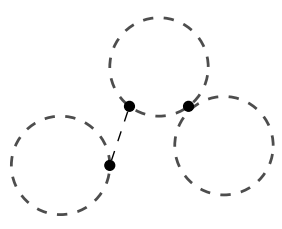

(j) $\alpha_{10}^{n}$

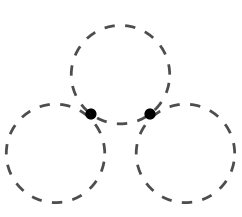

(k) $\alpha_{11}^{n}$
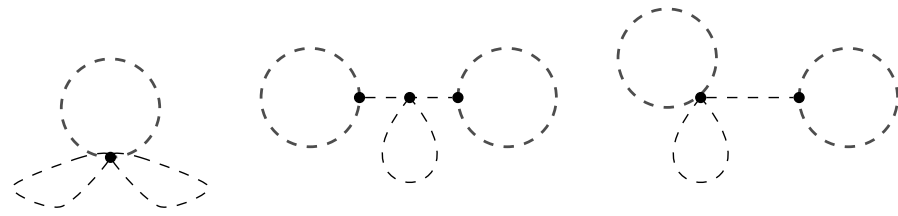

(n) $\alpha_{14}^{n}$

(o) $\alpha_{15}^{n}$

Fig. 10. Representation of tricyclic graphs in $\mathbb{X}_{0}^{n}$ which has no pendant vertices.

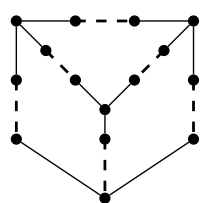

(a) $\beta_{1}^{n}$

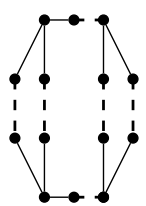

(b) $\beta_{2}^{2}$

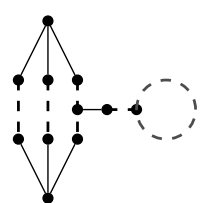

(c) $\beta_{3}^{n}$

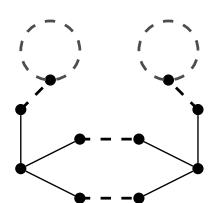

(d) $\beta_{4}^{n}$

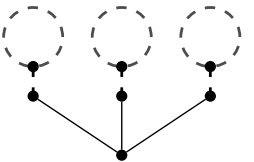

(e) $\beta_{5}^{n}$

Fig. 11. Tricyclic graphs in $\mathbb{X}_{1}^{n}$

\section{ACKNOWLEDGMENTS}

The research of Abhay Rajpoot is supported by CSIR-UGC JRF, New Delhi, India, through Reference No.: 1120/(CSIR-UGC NET DEC. 2016) and Lavanya Selvaganesh is financially supported by SERB, India, through Grant No. MTR/2018/000254 under the scheme MATRICS.

\section{References.}

Ali, A. \& Bhatti, A. A. (2016). A note on the augmented Zagreb index of cacti with fixed number of vertices and cycles, Kuwait Journal of Science, 43(4): 11-17.

Ali, A., Elumalai, S. \& Mansour, T. (2020). On the symmetric division deg index of molecular graphs, MATCH -Commun. Math. Comput. Chem., 83: 205-220.

Ali, A., Milovanović, E., Matejić, M. \& Milovanović, I. (2020). On the upper bounds for the degree deviation of graphs, Journal of Applied Mathematics and Computing, 62(1): 179-187.

Ali, A., Raza, Z. \& Bhatti, A. A. (2016). On the augmented Zagreb index, Kuwait Journal of Science, 43(2): 48-63.

Ali, A., Zhong, L. \& Gutman, I. (2019). Harmonic index and its generalizations: extremal results and bounds, MATCH -Commun. Math. Comput. Chem., 81: 249-311. 
Aouchiche, M., El Hallaoui, I. \& Hansen, P. (2020). Geometric-arithmetic index and minimum degree of connected graphs, MATCH -Commun. Math. Comput. Chem., 83(1): 179-188.

Basak, S. C. (2016). Use of graph invariants in quantitative structure-activity relationship studies, Croatica Chemica Acta, 89(4): 419-429.

Bondy, J. A., Murty, U. S. R. et al. (1976). Graph theory with applications (Vol. 290), Macmillan, London.

Borovicanin, B., Das, K. C., Furtula, B. \& Gutman, I. (2017). Bounds for Zagreb indices, MATCH -Commun. Math. Comput. Chem., 78(1): 17-100.

De, N. (2012). Some bounds of reformulated Zagreb indices, Appl. Match. Sci., 6(101): 5005-5012.

Deng, H. (2007). A unified approach to the extremal Zagreb indices for trees, unicyclic graphs and bicyclic graphs, MATCH -Commun. Math. Comput. Chem., 57(3): 597-616.

Devillers, J. \& Balaban, A. T. (2000). Topological indices and related descriptors in QSAR and QSPAR, CRC Press.

Fajtlowicz, S. (1987). On conjectures of graffiti II, Congr. Numer. 60: 187-197.

Furtula, B., Graovac, A. \& Vukičević, D. (2010). Augmented Zagreb index, Journal of mathematical chemistry, 48(2): 370-380.

Ghalavand, A. \& Ashrafi, A. R. (2017). Extremal trees with respect to the first and second reformulated Zagreb index, Malaya Journal of Matematik (MJM), 5(3): 524-530.

Ghalavand, A. \& Ashrafi, A. R. (2020). Ordering of c-cyclic graphs with respect to total irregularity, J. Appl. Math. Comput. 63: 707-715.

Gutman, I. (2013). Degree-based topological indices, Croatica Chemica Acta, 86(4): 351-361.

Gutman, I. \& Das, K. C. (2004). The first Zagreb index 30 years after, MATCH -Commun. Math. Comput. Chem., 50(1): 83-92.

Gutman, I., Furtula, B. \& Elphick, C. (2014). Three new/old vertex-degree-based topological indices, MATCH Commun. Math. Comput. Chem., 72(3): 617-632.

Gutman, I., Milovanović, E. \& Milovanović, I. (2020). Beyond the Zagreb indices, AKCE International Journal of Graphs and Combinatorics, 17(1): 74-85.

Gutman, I. \& Trinajstić, N. (1992). Graph theory and molecular orbitals. total $\phi$-electron energy of alternant hydrocarbons, Chemical Physics Letters, 17(4): 535-538.

Hosoya, H. (1971). Topological index. a newly proposed quantity characterizing the topological nature of structural isomers of saturated hydrocarbons, Bulletin of the Chemical Society of Japan, 44(9): 2332-2339.

Ilić, A. \& Zhou, B. (2012) . On reformulated Zagreb indices, Discrete Applied Mathematics, 160(3): 204-209.

Ji, S., Li, X. \& Huo, B. (2014). On reformulated Zagreb indices with respect to acyclic, unicyclic and bicyclic graphs, MATCH Commun. Math. Comput. Chem., 72(3): 723-732.

Ji, S., Qu, Y. \& Li, X. (2015). The reformulated Zagreb indices of tricyclic graphs, Applied Mathematics and Computation, 268: 590-595.

Karelson, M. (2000). Molecular descriptors in QSAR/QSPR, Wiley-Interscience. 
Khalifeh, M., Yousefi-Azari, H. \& Ashrafi, A. R. (2009). The first and second Zagreb indices of some graph operations, Discrete Applied Mathematics, 157(4): 804-811.

Klein, D. J., Lukovits, I. \& Gutman, I. (1995). On the definition of the hyper-wiener index for cyclecontaining structures, Journal of chemical information and computer sciences, 35(1): 50-52.

Miličević, A., Nikolić, S. \& Trinajstić, N. (2004). On reformulated Zagreb indices, Molecular diversity, 8(4): 393-399.

Narayankar, K. P., Kahsay, A. T. \& Klavzar, S. (2020). On peripheral wiener index: Line graphs, Zagreb index, and cut method, MATCH-Commun. Math. Comput. Chem., 83(1): 129-141.

Noureen, S., Ali, A. \& Bhatti, A. A. (2020). On the extremal Zagreb indices of n-vertex chemical trees with fixed number of segments or branching vertices, MATCH-Commun. Math. Comput. Chem., 84(2): $513-53$.

Rada, J. \& Bermudo, S. (2019). Is every graph the extremal value of a vertex-degree-based topological index, MATCH-Commun. Math. Comput. Chem., 81: 315-323.

Rajpoot, A. \& Selvaganesh, L. (2020). Bounds of the symmetric division deg index for trees and unicyclic graphs with a perfect matching, Iranian Journal of Mathematical Chemistry, 11(3): 141-159.

Randić, M. (1975). Characterization of molecular branching, Journal of the American Chemical Society, 97(23): 6609-6615.

Romero-Valencia, J., Hernández-Gómez, J. C. \& Reyna-Hernández, G. (2019). On the inverse degree index and decompositions in graphs, Kuwait Journal of Science, 46(4): 14-22.

Shang, Y. (2016). Estimating the distance Estrada index, Kuwait Journal of Science, 43(3): 14-19.

Su, G., Xiong, L., Xu, L. \& Ma, B. (2011). On the maximum and minimum first reformulated Zagreb index of graphs with connectivity at most $k$, Filomat, 25(4): 75-83.

Varmuza, K., Dehmer, M. \& Bonchev, D. (2012). Statistical modelling of molecular descriptors in QSAR/QSPR, Wiley Online Library.

Wiener, H. (1947). Structural determination of paraffin boiling points, Journal of the American Chemical Society, 69(1): 17-20.

Xu, K. \& Das, K. C. (2012). Trees, unicyclic, and bicyclic graphs extremal with respect to multiplicative sum Zagreb index, MATCH Commun. Math. Comput. Chem., 68(1): 257.

$\begin{array}{lr}\text { Submitted: } & 23 / 07 / 2020 \\ \text { Revised: } & 12 / 01 / 2021 \\ \text { Accepted: } & 07 / 02 / 2021 \\ \text { DOI: } 10.48129 / \text { kjs.v49i1.10447 }\end{array}$

